\title{
ROADMAP
}

CrossMark

\section{A roadmap for sole Cherenkov radiators with SiPMs in TOF-PET}

OPEN ACCESS

RECEIVED

20 January 2021

REVISED

19 August 2021

ACCEPTED FOR PUBLICATION

25 August 2021

PUBLISHED

21 September 2021

Original content from this work may be used under

the terms of the Creative

Commons Attribution 4.0

licence.

Any further distribution of this work must maintain

attribution to the

author(s) and the title of

the work, journal citation

and DOI.
Nicolaus Kratochwil ${ }^{1,2, *}$ (1), Stefan Gundacker ${ }^{1,3,4}$ (1) and Etiennette Auffray ${ }^{1}$

1 CERN, Esplanade des Particules 1, 1211 Meyrin, Switzerland

2 University of Vienna, Universitaetsring 1, A-1010 Vienna, Austria

3 Department of Physics of Molecular Imaging Systems, Institute for Experimental Molecular Imaging, RWTH Aachen University, Forckenbeckstrasse 55, D-52074 Aachen, Germany

4 Present address: Department of Physics of Molecular Imaging Systems, Institute for Experimental Molecular Imaging, RWTH Aachen University, Forckenbeckstrasse 55, 52074 Aachen, Germany.

* Author to whom any correspondence should be addressed.

E-mail: nicolaus.kratochwil@cern.ch

Keywords: $\mathrm{PbF}$, Cherenkov radiation, fast timing, silicon photomultiplier, time-of-flight PET, coincidence time resolution

\section{Abstract}

Time of flight positron emission tomography can strongly benefit from a very accurate time estimator given by Cherenkov radiation, which is produced upon a $511 \mathrm{keV}$ positron-electron annihilation gamma interaction in heavy inorganic scintillators. While time resolution in the order of $30 \mathrm{ps}$ full width at half maximum (FWHM) has been reported using MCP-PMTs and black painted Cherenkov radiators, such solutions have several disadvantages, like high cost and low detection efficiency of nowadays available MCP-PMTs. On the other hand, silicon photomultipliers (SiPMs) are not limited by those obstacles and provide high photon detection efficiency with a decent time response. Timing performance of $\mathrm{PbF}_{2}$ crystals of various lengths and surface conditions coupled to SiPMs was evaluated against a reference detector with an optimized test setup using high-frequency readout and novel time walk correction, with special attention on the intrinsic limits for one detected Cherenkov photon only. The average number of detected Cherenkov photons largely depends on the crystal surface state, resulting in a tradeoff between low photon time spread, thus good timing performance, and sensitivity. An intrinsic Cherenkov photon yield of $16.5 \pm 3.3$ was calculated for $2 \times 2 \times 3 \mathrm{~mm}^{3}$ sized $\mathrm{PbF}_{2}$ crystals upon $511 \mathrm{keV} \gamma$-deposition. After time walk correction based on the slew rate of the signal, assuming two identical detector arms in coincidence, and using all events, a time resolution of 215 ps FWHM (142 ps FWHM) was obtained for $2 \times 2 \times 20 \mathrm{~mm}^{3}\left(2 \times 2 \times 3 \mathrm{~mm}^{3}\right)$ sized $\mathrm{PbF}_{2}$ crystals, compared to $261 \mathrm{ps}$ (190 ps) without correction. Selecting on one detected photon only, a single photon coincidence time resolution of 113 ps FWHM for black painted and 166 ps for Teflon wrapped crystals was measured for $3 \mathrm{~mm}$ length, compared to 145 ps (black) and 263 ps (Teflon) for $20 \mathrm{~mm}$ length. 


\section{Contents}

1. Introduction 3

2. Material and methods $\quad 5$

3. Cherenkov photon yield 9

4. Time resolution results 11

5. Discussion on the results 16

6. Applicability of sole Cherenkov radiators with SiPMs at the system level 18

$\begin{array}{ll}7 . & \text { Outlook }\end{array}$

8. Conclusion 21 


\section{Introduction}

Positron emission tomography (PET) is presently the most sensitive molecular imaging modality, allowing to image the metabolic process in a patient and to detected diseases. One way of improving the PET performance is to establish time of flight (TOF), in order to reject random events and to improve the signal to noise ratio (SNR), for which the detectors need to have a very good time response (Conti and Bendriem 2019). Standard scintillation in crystals is defined by their rise time $\tau_{r}$, decay time $\tau_{d}$ and intrinsic light yield, which sets fundamental limits on the initial photon time density (Seifert et al 2012, Gundacker et al 2016a, Vinogradov 2018, Schaart 2021).

In TOF-PET hot electrons are produced upon $511 \mathrm{keV} \gamma$-interaction in the crystal, via photo-absorption or Compton scattering. These charged particles are passing through the crystal dielectric medium at a speed faster than the phase velocity of light and emit Cherenkov photons. In the timescale of standard scintillation emission these photons are considered as prompt, i.e. $\tau_{r} \approx \tau_{d} \approx 10 \mathrm{ps}$, and can serve as a very precise time tagger. In high energy physics, simultaneous measurement of Cherenkov and scintillation light can help to identify the electromagnetic fraction of the hadronic shower and therefore improve the energy resolution of hadron calorimeters (Lucchini et al 2020). The Cherenkov photon yield is largely dropping when approaching lower particle energies such as it is the case in prompt gamma imaging for range verification in hadron therapy (ArinoEstrada et al 2019) and TOF-PET. For the latter the detection of few prompt photons on top of scintillation can largely improve the timing performance (Gundacker et al 2016a, Kratochwil et al 2020a) and is one way toward the achievement of the 10 ps time resolution target (Lecoq et al 2020). Such unprecedented time resolutions can largely improve the performance of TOF-PET scanners allowing faster medical examinations, dose reduction, better image quality and might open the door to real-time-imaging and prenatal diagnosis. Beside aiming for the best possible timing performance, Cherenkov photons can also be utilized to enable more affordable TOF-PET scanners by optimizing sensitivity and cost (Brunner and Schaart 2017, Kratochwil et al 2020b), since crystals with lower melting temperature and cheaper raw materials like $\mathrm{BGO}$ or $\mathrm{PbF}_{2}$ can be used.

Using pure Cherenkov radiators without scintillation, and sacrificing energy resolution in TOF-PET, was already proposed more than 10 years ago (Dolenec et al 2011). Recently coincidence time resolution (CTR) values as good as $30 \mathrm{ps}$ full width at half maximum (FWHM) for a small fraction of events were reported by using Cherenkov-radiator-integrated MCP-PMTs (Ota et al 2019), although with non-satisfying sensitivity. Looking only at the best achievable time resolution, the combination of MCP-PMTs with black painted pure Cherenkov radiators (Ota et al 2021) outperform all known scintillating inorganic crystals coupled to silicon photomultipliers (SiPMs) (Gundacker et al 2020a). However, despite the excellent timing properties of MCPPMTs, there are several drawbacks like high cost, low tolerance to magnetic field, bulky size, aging and low quantum efficiency of the photocathode. In particular the last is of utmost importance in the case of few produced Cherenkov photons, since events where no photons are detected are lost, resulting in a reduction of PET detector sensitivity. At the same time a low detection probability translates in worse capability of discriminating low energy scattered gammas from unscattered $511 \mathrm{keV}$ gammas, inevitable making the image reconstruction procedure more difficult.

SiPMs (Gundacker and Heering 2020) can provide a flexible and cost effective choice of photodetectors for pure Cherenkov radiators, as they are not limited by the drawbacks mentioned above for MCP-PMTs. Their higher photon detection efficiency with respect to MCP-PMTs can compensate the worse timing properties allowing a similar effective detector sensitivity (PET detector gamma detection efficiency/CTR) (Schaart et al 2021) when considering pure Cherenkov based gamma detectors.

The use of SiPMs with lead(II)fluoride $\left(\mathrm{PbF}_{2}\right)$ crystals has first been evaluated by Dolenec et al (2015) with moderate CTR above 400 ps FWHM and a good SNR, when the SiPMs are cooled to reduce their usually high dark count rate (DCR). However, the detector chain (SiPM + electronics + digitization) was not optimized to exploit all SiPM capabilities. This value was shown to improve to 197 ps FWHM for $15 \mathrm{~mm}$ long crystal when selecting on one single photon avalance diode (SPAD) events (Consuegra et al 2019). Simulation studies (Consuegra et al 2020) showed, that for a hypothetical 0 ps single photon time resolution (SPTR) of the SiPM, CTRs as good as 22 ps FWHM can be reached when the crystals are small and black painted. Similar values (30 ps) are reported in Gundacker et al (2020a) for BGO with 10 ps SPTR utilizing Cherenkov photons. In ArinoEstrada et al (2021) SiPMs were used to study the timing capabilities of semiconductor crystals and a statistical approach was used to estimate the mean number of detected Cherenkov photons, however without considering the rather high optical crosstalk in SiPMs. After reference correction and selection on more than seven triggered SPADs about 400 ps FWHM were reported for TlCl crystals. In general the use of semiconductor crystals/ perovskites like $\mathrm{TlBr}, \mathrm{TlCl}, \mathrm{Cs} \mathrm{PbBr}_{3}$ or $\mathrm{CsPbCl}_{3}$ are promising Cherenkov radiators due to the capability of utilizing excellent energy resolution with charge readout (Kim et al 2009, Tao et al 2019, Arino-Estrada et al 2020). 
In this study the timing capabilities for pure Cherenkov radiators using $\mathrm{PbF}_{2}$ with state-of-the-art $\mathrm{SiPMs}$ and high frequency (HF) electronics (Cates et al 2018, Gundacker et al 2019) were evaluated. A statistical model (Vinogradov 2012) including crosstalk has been used to evaluate the Cherenkov photon yield for various surface conditions, crystal lengths and as a function of the direction of the readout with respect to the incoming gamma. Depth of interaction (DOI) collimated measurements were performed to resolve the propagation of photons inside the crystal. The article is structured as followed: in section 2 the performed measurements, analysis, theoretical background and time walk correction are described. Section 3 presents the measured SiPM crosstalk and Cherenkov photon yield. In section 4 time resolution results are presented with considerations of the intrinsic limits. In the sections 5-7 the results are discussed and thoughts on the applicability on a system level and insights for future improvements are given. 


\section{Material and methods}

\section{1. $\mathrm{PbF}_{2}$ crystals}

The crystals used for this study were produced by Epic-Crystals and were fully polished. The tested crystals had a cross section of $2 \times 2 \mathrm{~mm}^{2}$ and a length ranging from 3 to $30 \mathrm{~mm}$. Depending on the measurement conditions, the crystals were either kept as they are (Bare), wrapped with more than 5 layers of Teflon (Teflon), black painted using black spray (LUXENS) with refractive index of $n=1.5$ (Black) or a combination using enhanced specular reflector (ESR) opposite to the readout, as summarized in table 1, before optically coupling to the SiPMs with Cargille Meltmount ( $n=1.582$ ). The Cherenkov emission probability follows a $1 / \lambda^{2}$ distribution, so that more photons are produced at shorter wavelength. $\mathrm{PbF}_{2}$ is in principle transparent up to around $250 \mathrm{~nm}$, but the coupling agent and the used SiPMs only allowed to use the emission up to $300 \mathrm{~nm}$.

For one measurement a quartz $\left(\mathrm{SiO}_{2}\right)$ glass $\left(2 \times 2 \times 4 \mathrm{~mm}^{3}\right.$, black painted $)$ was tested. This pixel was cut from a larger piece and kept unpolished.

One advantage of $\mathrm{PbF}_{2}$ compared to other inorganic scintillating crystals is the low cost of the row materials and moderate melting point, yielding to a cost effective crystal solution. In addition, $\mathrm{PbF}_{2}$ features high effective atomic number and high density, yielding to short gamma attenuation length or high stopping power. Key properties of $\mathrm{PbF}_{2}$ and other well known materials are summarized in table 2.

\subsection{Silicon photomultiplier}

For all measurements NUV-HD SiPMs from fondazione bruno kessler (FBK) were used, having a cross section of $4 \times 4 \mathrm{~mm}^{2}$ and $40 \mu \mathrm{m}^{2}$ SPAD pitch and no protective resin window. The performance of these devices was extensively studied in the past (Gundacker et al 2016b, Gola et al 2019) and holds the current record in terms of excellent intrinsic SPTR of $69 \pm 6$ ps FWHM and CTR of $58 \pm 3$ ps FWHM for small LSO:Ce:Ca crystals (Gundacker et al 2019). The same configuration (LSO + FBK) was used as reference detector, but with a slightly different photopeak region for better statistics.

\subsection{CTR setup}

The CTR was measured with the setup described in Gundacker et al (2019) in a temperature stabilized environment $\left(\approx 18^{\circ} \mathrm{C}\right) . \mathrm{A}^{22} \mathrm{Na}$ source with an activity of $3 \mathrm{MBq}$ emits two gammas back to back which were detected in coincidence by the reference detector $\left(2 \times 2 \times 3 \mathrm{~mm}^{3} \mathrm{LSO}\right.$ :CeCa, CTR $\left.=61.2 \pm 3.0 \mathrm{ps} \mathrm{FWHM}\right)$ and Cherenkov radiator under test. For the reference detector the signal was split, where one part was amplified using HF electronics for the time signal and the other part via an analog operational amplifier (AD8000) for the energy signal. The signals were digitized with a LeCroy DDA735Zi oscilloscope, having a bandwidth of $3.5 \mathrm{GHz}$ and sampling rate of $20 \mathrm{Gs} \mathrm{s}^{-1}$. For the other detector side $\left(\mathrm{PbF}_{2}\right)$ only the HF signal was used, and the amplitude and charge of the HF signal was measured within a time window of $5 \mathrm{~ns}$ around the signal. Moreover the slew rate $(\mathrm{d} V / \mathrm{d} t)$ at different threshold positions $(5,20,30 \mathrm{mV})$ and the signal rise time $(5-20 \mathrm{mV})$ were recorded. The single SPAD signal amplitude at operational condition (39 V bias voltage) was $44 \mathrm{mV}$, while the time difference was calculated via leading edge discrimination at $10 \mathrm{mV}$. All time resolution values were corrected for the reference detector contribution, assuming two identical Cherenkov radiators in coincidence. The duration of one measurement condition (SiPM overvoltage, leading edge threshold, DOI position) changed between one hour and two days, but it was ensured that at least 10k coincidence events were acquired after photopeak selection of the reference detector.

An illustration of the setup is sketched on the left of figure 1, while on the right a screen-shot of the oscilloscope with typical detector pulses is depicted.

Measurements presented in section 4.4 were performed in a DOI collimated configuration where the reference crystal was placed at a distance of $60 \mathrm{~mm}$ and the source of $10 \mathrm{~mm}$ from the $\mathrm{PbF}_{2}$ crystal. The crystal was irradiated from the lateral side and moved vertically for different DOI measurements.

The used custom made electronics is fully described in Cates et al (2018), Gundacker et al (2019) to have low influence of electronic noise, which is of utmost importance to achieve excellent time resolution, particular for low light intensities (Cates and Levin 2019, Kratochwil et al 2020a, Gundacker et al 2020a). A 3 GHz balun transformer and two BGA616 bipolar monolithic microwave integrated circuit amplifiers with $570 \mathrm{~mW}$ total power consumption were used to obtain the time response of a single channel.

\subsection{Cross talk evaluation}

The number of detected Cherenkov photons is very low in the order of few triggered SPADs only and not distinguishable from random triggered dark count events ( $\leqslant 5$ counts per $\mu$ s (Gola et al 2019)). Crosstalk happens when secondary photons are produced during the SPAD avalanche process, which subsequently are detected by another SPAD. The crosstalk probability was deduced with the same setup as for the CTR 
Table 1. Summary of performed measurement conditions. DOI collimated measurements were only performed for some 20 and $30 \mathrm{~mm}$ long surface conditions while all 3 orientations (default head on irradiation, side irradiation and back irradiation with SiPM between source and Cherenkov radiator) were tested for one configuration.

\begin{tabular}{|c|c|c|c|c|}
\hline Material & $\begin{array}{c}\text { Geometry } \\
\left(\mathrm{mm}^{3}\right)\end{array}$ & $\begin{array}{l}\text { Lateral } \\
\text { surface }\end{array}$ & $\begin{array}{c}\text { Back } \\
\text { surface }\end{array}$ & Orientation \\
\hline $\mathrm{PbF}_{2}$ & $2 \times 2 \times 3$ & Teflon & Teflon & Head-on \\
\hline $\mathrm{PbF}_{2}$ & $2 \times 2 \times 3$ & Bare & Bare & Head-on \\
\hline $\mathrm{PbF}_{2}$ & $2 \times 2 \times 3$ & Black & Black & Head-on \\
\hline $\mathrm{PbF}_{2}$ & $2 \times 2 \times 3$ & Teflon & Black & $\begin{array}{c}\text { All } 3 \\
\text { orientations }\end{array}$ \\
\hline $\mathrm{PbF}_{2}$ & $2 \times 2 \times 5$ & Black & Black & Head-on \\
\hline $\mathrm{PbF}_{2}$ & $2 \times 2 \times 10$ & Black & Black & Head-on \\
\hline $\mathrm{PbF}_{2}$ & $2 \times 2 \times 15$ & Black & Black & Head-on \\
\hline $\mathrm{PbF}_{2}$ & $2 \times 2 \times 20$ & Black & Black & $\begin{array}{l}\text { Head-on } \\
+ \text { DOI }\end{array}$ \\
\hline $\mathrm{PbF}_{2}$ & $2 \times 2 \times 30$ & Black & Black & Head-on \\
\hline $\mathrm{PbF}_{2}$ & $2 \times 2 \times 20$ & Teflon & Teflon & $\begin{array}{l}\text { Head-on } \\
+ \text { DOI }\end{array}$ \\
\hline $\mathrm{PbF}_{2}$ & $2 \times 2 \times 20$ & Teflon & Black & Head-on \\
\hline $\mathrm{PbF}_{2}$ & $2 \times 2 \times 30$ & Black & ESR & DOI \\
\hline Quartz & $2 \times 2 \times 4$ & Black & Black & Head-on \\
\hline
\end{tabular}

Table 2. Physical properties of selected Cherenkov radiators and inorganic scintillators.

\begin{tabular}{|c|c|c|c|c|c|c|c|c|}
\hline Material & $\mathrm{PbF}_{2}$ & $\mathrm{~B}_{4} \mathrm{Ge}_{3} \mathrm{O}_{12}$ & $\mathrm{SiO}_{2}$ & $\mathrm{TlBr}$ & $\mathrm{TlCl}$ & $\mathrm{HfO}_{2}$ & $\mathrm{Lu}_{2} \mathrm{SiO}_{5}: \mathrm{Ce}$ & $\mathrm{PbWO}_{4}$ \\
\hline Density $\left(\mathrm{g} \mathrm{cm}^{-3}\right)$ & $7.8^{\mathrm{a}}$ & $7.1^{\mathrm{g}}$ & $2.7^{\mathrm{a}}$ & $7.5^{\mathrm{f}}$ & $7^{\mathrm{f}}$ & $9.7^{\mathrm{h}}$ & $7.3^{g}$ & $8.3^{\mathrm{g}}$ \\
\hline$Z_{\text {eff }}$ & $77^{\mathrm{b}}$ & $71^{\mathrm{b}}$ & $12^{\mathrm{b}}$ & $73^{b}$ & $76^{\mathrm{b}}$ & $67^{\mathrm{b}}$ & $64^{\mathrm{b}}$ & $74^{\mathrm{b}}$ \\
\hline Attenuation length (mm) & $8.7^{\mathrm{c}}$ & $10.1^{c}$ & $42.4^{\mathrm{c}}$ & $9.7^{c}$ & $9.7^{\mathrm{c}}$ & $8.2^{\mathrm{c}}$ & $11.4^{\mathrm{c}}$ & $8.5^{\mathrm{c}}$ \\
\hline Cutoff wavelength (nm) & $250^{\mathrm{d}}$ & $300^{\mathrm{d}}$ & $170^{g}$ & $440^{\mathrm{f}}$ & $380^{\mathrm{f}}$ & $200^{\mathrm{h}}$ & $370^{\mathrm{d}}$ & $320^{\mathrm{d}}$ \\
\hline Ref. ind. $n$ at $550 \mathrm{~nm}$ & $1.77^{\mathrm{e}}$ & $2.12^{\mathrm{e}}$ & $1.46^{\mathrm{e}}$ & $2.47^{\mathrm{e}}$ & $2.28^{\mathrm{e}}$ & $1.9^{\mathrm{e}}$ & $1.8^{\mathrm{e}}$ & $2.16^{\mathrm{e}}$ \\
\hline Peak emission (nm) & $1 / \lambda^{2}$ & $480^{g}$ & $1 / \lambda^{2}$ & $1 / \lambda^{2}$ & $1 / \lambda^{2}$ & $1 / \lambda^{2}$ & $420^{g}$ & $420^{\mathrm{g}}$ \\
\hline Melting point $\left({ }^{\circ} \mathrm{C}\right)$ & $824^{\mathrm{a}}$ & $1050^{\mathrm{f}}$ & $1610^{\mathrm{a}}$ & $460^{\mathrm{f}}$ & $430^{\mathrm{a}}$ & $2812^{\mathrm{a}}$ & $2050^{\mathrm{f}}$ & $1123^{g}$ \\
\hline
\end{tabular}

a GESTIS database, https://gestis.dguv.de/data.

b Approximation calculated according to Murty (1965).

c At 500 keV, NIST database, https://physics.nist.gov/cgi-bin/Xcom/xcom2.

${ }^{\mathrm{d}}$ Measured.

e Refractive index database, https://refractiveindex.info/.

${ }^{\mathrm{f}}$ Arino-Estrada et al (2021).

g Epic Crystal datasheet, https://www.epic-crystal.com/.

${ }^{\mathrm{h}}$ Ota et al (2020).

measurements, but without the radioactive ${ }^{22} \mathrm{Na}$ source in the test bench and by triggering only on the HF signal of the SiPM coupled to the crystal. A histogram of the measured charge was drawn and the number of triggered SPADs $\left(N_{1}, N_{2}, \ldots N_{k}\right)$ were counted. Because of a very small probability of two dark count events appearing in the same time window, it can be assumed with certainty that all events above one triggered SPAD are due to crosstalk. The timescale of delayed crosstalk and after-pulsing was well above the used $5 \mathrm{~ns}$ time window (Acerbi and Gundacker 2019). To model the distribution of triggered SPADs a branching Poisson process (Borel $\lambda$ ) was used (Vinogradov 2012), because a simple geometric chain is not accurate in the case of high crosstalk probabilities. Equation (1) denotes the probability $P_{k, D C R}$ of measuring the signal of $k$ triggered SPADs after the initial dark count event, as function of the crosstalk parameter $\lambda$.

$$
P_{k, D C R}=\frac{(\lambda \cdot k)^{k-1} \cdot \exp (-k \cdot \lambda)}{k !} \quad \text { for } k \geqslant 1
$$

The case $k=1$ describes the probability of not having crosstalk while $1-P_{k=1, D C R}$ is the probability of having one or more SPADs triggered due to crosstalk. $\lambda$ is extracted via chi-square minimization (Berkson 1980) using the measured values $N_{k}$ by $P_{k, D C R}=\frac{N_{k}}{\sum_{i \geqslant 1} N_{i}}$ and the conversion to the geometric model can be done via $p_{\text {geom }}=1-\exp (-\lambda)$. 


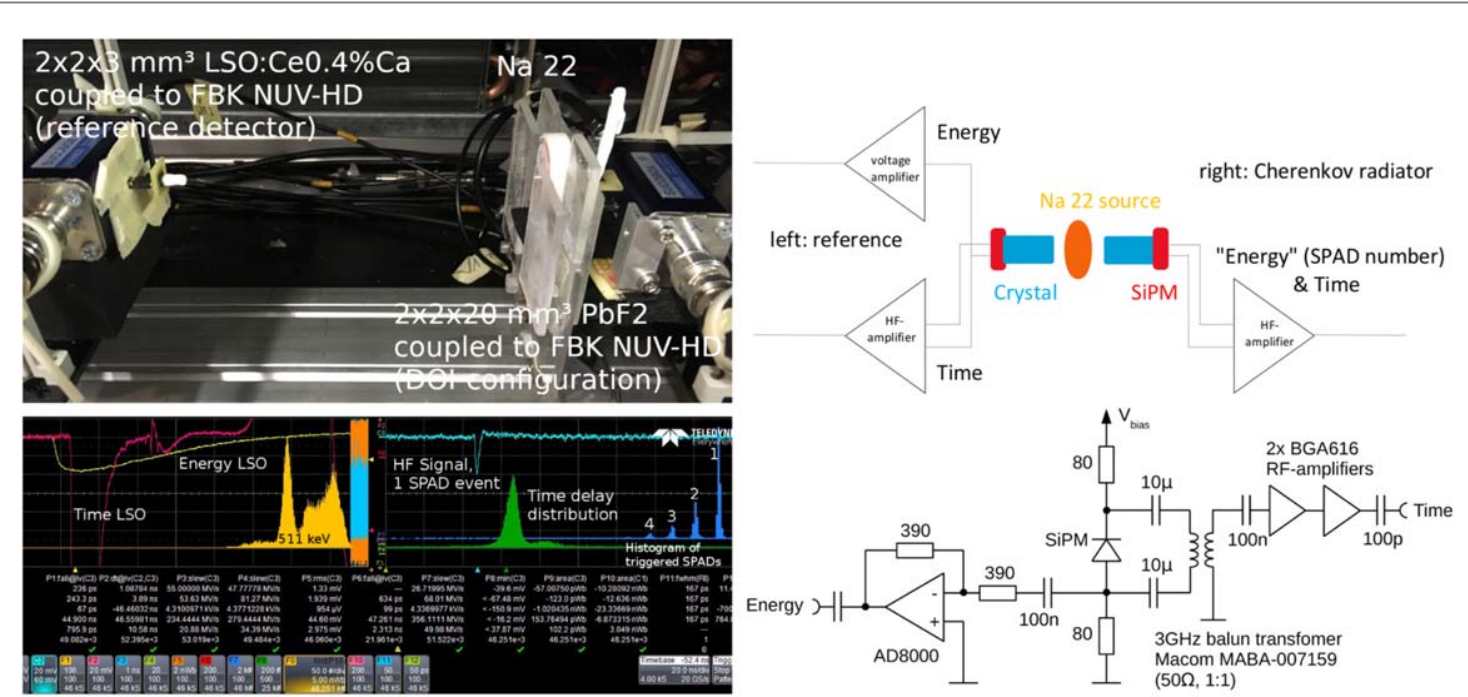

Figure 1. (top left) Picture of the CTR setup with reference detector and $\mathrm{PbF}_{2}$ crystal. The ${ }^{22} \mathrm{Na}$ source is placed close to the $\mathrm{PbF}$ crystal to ensure a depth-of-interaction collimated measurement. (top right) Schematics of the CTR setup: $\mathrm{a}^{22} \mathrm{Na}$ source is placed between the reference crystal and Cherenkov radiator under test. For the reference detector both energy and time information were used, while for the Cherenkov radiator solely the high frequency (time) signal was used to extract the coincidence time difference, number of triggered SPADs and SiPM signal slew rate. (bottom left) Screen-shot of the oscilloscope having the signal of the reference detector on the left with the typical ${ }^{22} \mathrm{Na}$ energy spectrum (reverse, due to negative signal) and on the right the signal of the Cherenkov radiator. The histogram in green represents the coincidence time delay without any event selection, while the finger plot in blue is coming from different number of triggered SPADs. (bottom right) Schematics of the high-frequency electronics, Reproduced from Gundacker et al (2019). (c) 2019 Institute of Physics and Engineering in Medicine. All rights reserved. SiPM anode and cathode are connected with pins to the electronics.

\subsection{Cherenkov photon yield estimation}

To estimate the mean $\mu$ number of detected Cherenkov photons a generalized Poisson $(\mu, \lambda)$ (Vinogradov 2012) was used, where the probability $P_{k}$ of having $k$ triggered SPADs was calculated as in equation (2). This distribution allows to have zero triggered SPADs (no photons detected) which are not measured. Therefore a new distribution shown in equation (3) was constructed by dividing over the probability of one-SAPD events to enable the extraction of $\mu$ using the measured crosstalk parameter $\lambda$, again via chi-square minimization.

$$
\begin{aligned}
& P_{k}=\frac{\mu \cdot(\mu+\lambda \cdot k)^{k-1} \cdot \exp (-\mu-k \cdot \lambda)}{k !} \text { for } k \geqslant 0 \\
& \tilde{P}_{k}=\frac{P_{k}}{P_{1}}=\frac{N_{k}}{N_{1}}=\frac{(\mu+\lambda \cdot k)^{k-1} \cdot \exp (\lambda-k \cdot \lambda)}{k !} \quad \text { for } k \geqslant 2 .
\end{aligned}
$$

From the estimated mean Cherenkov photon number $\mu$ the probability of detecting one or more photons $(k \geqslant 1)$ in coincidence can be calculated according to equation $(4)$, since the probability for $P_{0}$ is the simplified expression $\exp (-\mu)$

$$
P_{k \geqslant 1, \text { coinc }}=(1-\exp (-\mu))^{2} .
$$

The square in equation (4) is required when assuming two identical detectors, as for both detector arms more than zero photons need to be detected in coincidence.

\subsection{Modeling the time delay distribution and time walk correction}

The measured time delay distribution shows, in dependency of the condition more or less, a pronounced tail coming from photons which are bouncing long time in the crystal before being detected. For standard scintillation these tails are not that pronounced, as the trigger is on the first few detected photons and very late photons do not contribute. In the case of pure Cherenkov radiators, in several cases the first detected photon is also the last one. To account for this tail a Crystal Ball function, which consists of a Gaussian core portion and a power-law low-end tail, was used. The FWHM and full width at tenth maximum (FWTM) of the fit function was calculated and corrected for the reference contribution. When the distance between the Cherenkov radiator and the ${ }^{22} \mathrm{Na}$ source was large, a constant floor of events in the time delay distribution was observed coming from DCR. For reasons of consistency for all the measurements a constant term was added to the fit function to account for those events.

A strong time walk was observed depending on the number of detected photons, similar as reported in Dolenec et al (2016b). Time walk correction based on the amplitude or charge were tested with certain success, 
but clearly better performance was achieved when corrections based on the measured slew rate (SR) were made (see sections 4.1, 5.1 and table 4). A similar correction based on the signal rise time is described in the case of BGO in Kratochwil et al (2020b). 


\section{Cherenkov photon yield}

\subsection{Enhanced optical crosstalk due to crystal coupling}

The left of figure 2 shows the measured probability of triggering one, two and more SPADs due to the initial dark count event and a following cascade of crosstalk for different bias voltages and with Teflon wrapping or black painted crystals. On the right of figure 2 the calculated crosstalk parameter $\lambda$ as function of the bias voltage is drawn for all tested surface conditions for $2 \times 2 \times 3 \mathrm{~mm}^{3}$ sized crystals. We observed a higher crosstalk probability when the crystal is wrapped in Teflon, as it behaves like a reflector for the secondary photons generated by each avalanche. The lowest crosstalk parameter was measured for a black painted crystals and for black paint opposite to the readout side, since in this case most of the created avalanche photons are absorbed and do not contribute. The change of the correlated noise when coupling a crystal to the SiPM is in agreement with previous measurements using different crystal cross sections (Gundacker et al 2016b).

\subsection{Detected Cherenkov photons}

A histogram of the measured SiPM signal charge for $20 \mathrm{~mm}$ long crystals is presented on the left of figure 3 . While the black painted crystal had mostly one or two SPAD events and the occurrence droped very fast for higher SPAD number, it was less steep when the crystal was wrapped in Teflon. When counting the events having $k$ SPADs triggered and using the calculated crosstalk parameter and equation (3) the mean number of detected Cherenkov photons was evaluated, as shown on the right of figure 3. The calculated mean Cherenkov photon number $\mu$ denotes all events including cases where the $511 \mathrm{keV}$ gamma only deposited a fraction of its energy in the crystal. For larger crystal volumes (e.g. $4 \times 4 \times 30 \mathrm{~mm}^{3}$ instead of $2 \times 2 \times 3 \mathrm{~mm}^{3}$ ) on average more energy is deposited in the crystal (due to a higher fraction of Compton events being contained in the crystal) and therefore also more Cherenkov photons are produced and detected (see section 5.2). Similarly higher Cherenkov photon numbers are expected when selecting on photopeak events only (Arino-Estrada et al 2018).

Results in terms of crosstalk, Cherenkov photon yield and probability $P_{\text {coinc }}$ of detecting an event in coincidence are summarized in table 3.

\subsection{Directionality}

Based on simulations with Geant4, a weak directionality of the Cherenkov emission was expected (Brunner et al 2014, Roncali et al 2019). In particular when a Compton interaction occurs, the momentum of the recoil electron depends on the direction of the incident $\gamma$-ray and therefore also the Cherenkov emission cone. To validate this assumption the $2 \times 2 \times 3 \mathrm{~mm}^{3}$ sized crystal was black painted opposite to the readout side and wrapped in Teflon on the lateral faces to enhance the expected effect. The $\mathrm{PbF}_{2}$ crystal was placed far away $(\approx 15 \mathrm{~cm})$ from the reference crystal and the ${ }^{22} \mathrm{Na}$ source to have an almost parallel beam of gammas without precise alignment. The system of crystal, SiPM and amplifier was rotated so that irradiation from three different
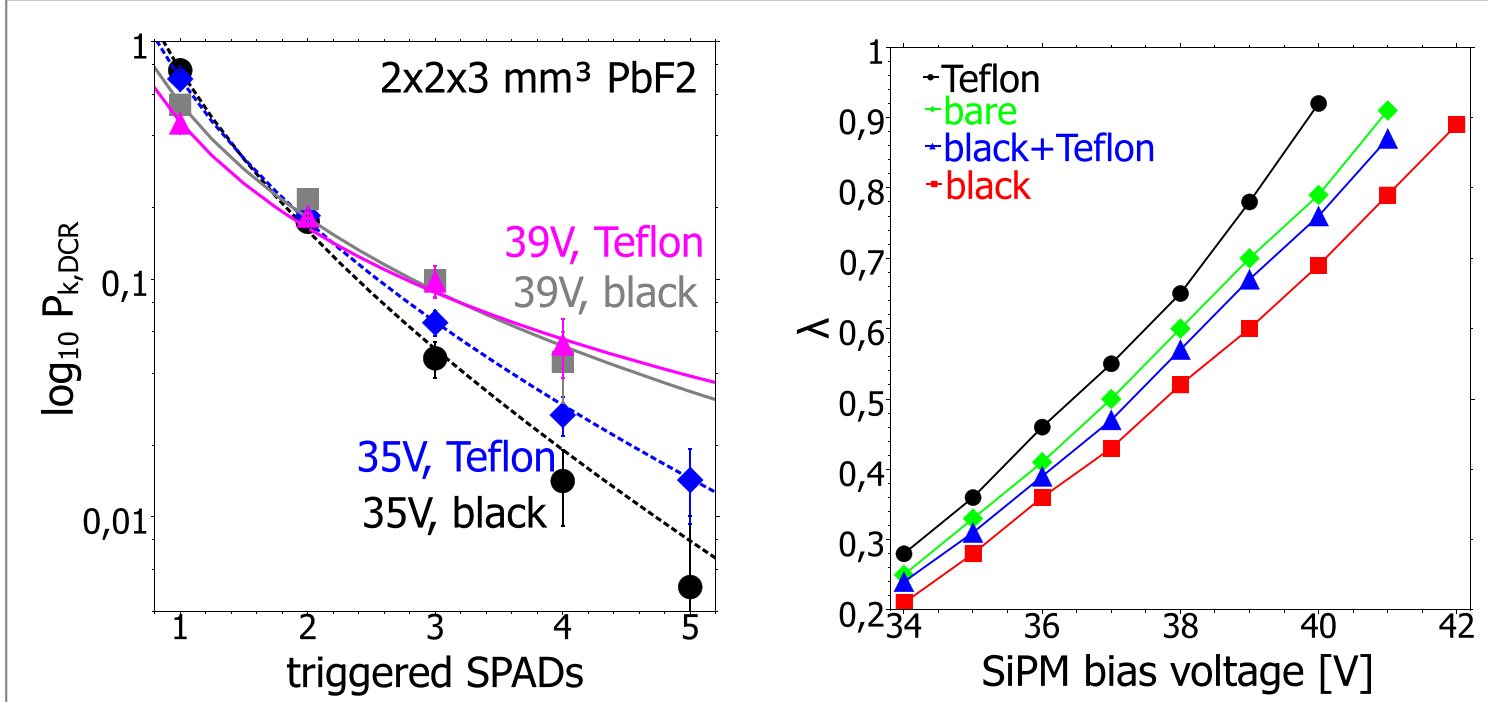

Figure 2. (left) Measured probability of having $k$ SPADs triggered for Teflon wrapped and black painted crystals including the fit function from equation (1). (right) Crosstalk parameter $\lambda$ as function of SiPM bias voltage and surface treatment. 

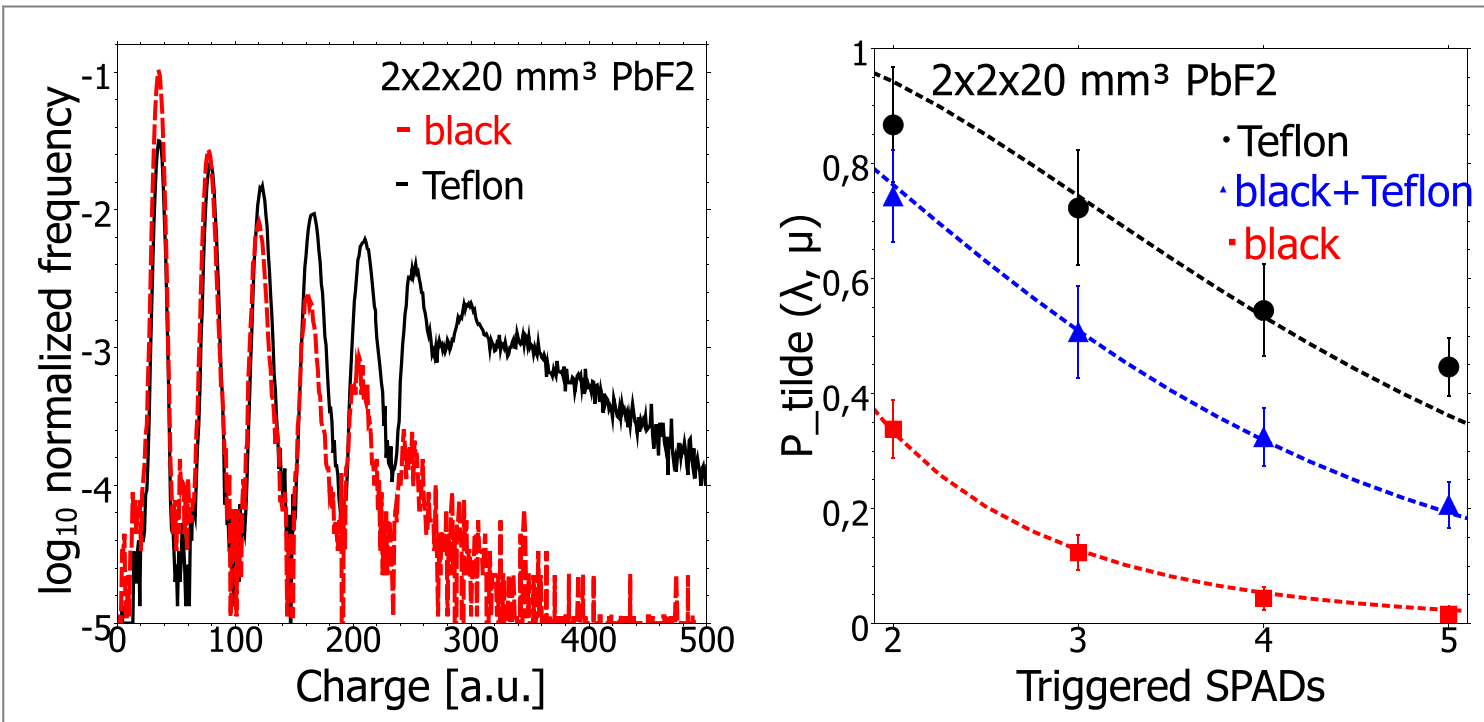

Figure 3. (left) Normalized histogram of the measured charge spectrum at $35 \mathrm{~V}$ bias voltage. The photoelectron resolution drastically reduces above five triggered SPADs since the SiPM signal exceeds the used range of the oscilloscope. (right) Re-normalized probability based on equation (3) of having $k$ SPADs triggered including the fit function for $20 \mathrm{~mm}$ long crystals.

Table 3. Crosstalk parameter and mean number of detected Cherenkov photons for various crystal configurations. The error of $\lambda$ is \pm 0.02 and for $\mu \pm 10 \%$, while $P_{\text {coinc }}$ is derived from $\mu$.

\begin{tabular}{lcccc}
\hline $\begin{array}{l}\text { Surface } \\
\text { condition }\end{array}$ & $\begin{array}{c}\text { Geometry } \\
\left(\mathrm{mm}^{3}\right)\end{array}$ & $\lambda @ 35 \mathrm{~V}$ & $\mu$ & $P_{\text {coinc }}(\%)$ \\
\hline Teflon & $2 \times 2 \times 3$ & 0.36 & 2.08 & $77_{-5}^{+3}$ \\
Bare & $2 \times 2 \times 3$ & 0.33 & 1.86 & $71_{-5}^{+5}$ \\
Black+Teflon & $2 \times 2 \times 3$ & 0.31 & 1.74 & $68_{-5}^{+5}$ \\
Black & $2 \times 2 \times 3$ & 0.28 & 0.71 & $26_{-4}^{+3}$ \\
Teflon & $2 \times 2 \times 20$ & 0.32 & 1.95 & $74_{-6}^{+4}$ \\
Black+Teflon & $2 \times 2 \times 20$ & 0.3 & 1.46 & $59_{-6}^{+5}$ \\
Black & $2 \times 2 \times 20$ & 0.28 & 0.32 & $7_{-1}^{+2}$ \\
Quartz black & $2 \times 2 \times 4$ & 0.29 & 0.49 & $15_{-2}^{+2}$ \\
\hline
\end{tabular}

orientations with respect to the readout side were possible (head on, side, back), as illustrated in figure 4. A minor drop to $94 \pm 6 \%$ of $\mu$ with respect to classical head-on condition for the side irradiation was calculated, but for back irradiation this value drops to $54 \pm 6 \%$ indicating a strong directionality of the emission. 
$2 \times 2 \times 3 \mathrm{~mm}^{3}$ LSO

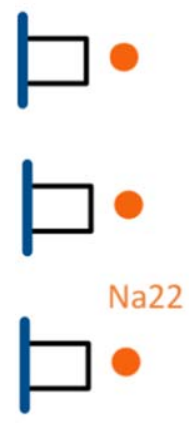

$2 \times 2 \times 3 \mathrm{~mm}^{3} \mathrm{PbF} 2$

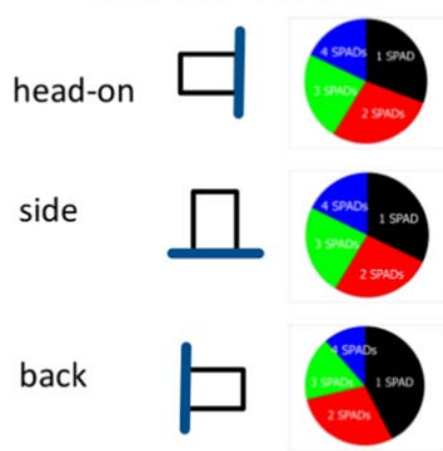

Figure 4. Illustration of the measurement configurations on directionality. The $\mathrm{PbF}_{2}$ crystal glued to the SiPM was rotated to irradiate it from different directions. The pie chart illustrates the relative distribution of one- (black), two- (red), three- (green) and four- (blue) SPAD events after dark count subtraction.

\section{Time resolution results}

\subsection{Time walk correction}

When selecting on coincidence events, having a photopeak on the reference crystal, the time delay distribution shows a non-Gaussian structure and a tail coming from the fluctuating number of triggered SPADs. The excellent single photon counting capability of the SiPM allows to select on events with different number of triggered SPADs, as illustrated on the top of figure 5. The peak of the distribution using all events is moved to the left compared to one, two or three triggered SPADs and more pronounced for the Teflon condition. The reason for this time walk is coming from a steeper signal which passes the leading edge discrimination faster than for a low number of triggered SPADs. A similar observation is reported by Liu et al (2016) for low light intensity detection with a Phillips digital SiPM.

The ratio of all events to one to three triggered SPADs is greater for the Teflon case than for the Black condition, since the average number of detected Cherenkov photons is larger in the first case leading to a different distribution of activated SPADs.

On the bottom left a scattered plot of the coincidence time delay $t$ against the slew rate $S R$ is shown. A polynomial function $f(S R)$ was used to fit the data and to correct the individual time stamps $t_{c o r, i}=t_{i}-f(S R)$. When drawing a histogram of the slew rate, peaks coming from one, two, and more triggered SPADs are visible (see section 5.1). The time delay distribution after correction is shown on the bottom right of figure 5. A large improvement of the time resolution after correction, in particular with respect to the tails is observed.

\subsection{Impact on the time resolution of crystal surface treatment and length}

The measured time delay distribution for $20 \mathrm{~mm}$ long crystal length is shown in figure 6 for all three tested surface conditions.

The measured time resolutions for all configurations are summarized in table 4.

Single photon coincidence time resolution (SPCTR) denotes events where only one SPAD was triggered. This way the photon time spread (PTS) contribution can be estimated for different surface contributions and lengths. Comparing Teflon with Black, an additional CTR contribution of $\approx 86 \pm 6$ ps FWHM (single side, 122 ps in coincidence, $86=\sqrt{166^{2}-113^{2}} / \sqrt{2}$ ) for small and $\approx 155 \pm 9 \mathrm{ps}$ for long crystals is observed in Teflon. This is due to a larger PTS in the crystal when wrapped in Teflon as compared to the black painted case, because of multiple reflections and scattering at the surface of the crystal.

Moreover, comparing Teflon wrapped $\mathrm{PbF}_{2}$ and $\mathrm{BGO}$, having a similar refractive index and a similar Cherenkov photon yield, the CTR is better for $\mathrm{PbF}_{2}\left(2 \times 2 \times 3 \mathrm{~mm}^{3}: 142 \pm 5 \mathrm{ps} \mathrm{FWHM} \mathrm{PbF}_{2}, 151 \pm 3 \mathrm{ps}\right.$ $\mathrm{BGO} / 2 \times 2 \times 20 \mathrm{~mm}^{3}: 215 \pm 8 \mathrm{ps} \mathrm{PbF}_{2}, 259 \pm 3$ ps BGO). This confirms, that slow scintillation photons lead to a deterioration of the time resolution, since they are not promptly produced and lead to a broadening of the time delay distribution when the initial Cherenkov photons are not detected. This is the price paid for a higher detection efficiency, since in the case of sole Cherenkov radiators these events are not detected.

It is interesting to notice that SPCTR is better than CTR after correction for crystals having low $\mu$ with Black configuration, while for Teflon high number of detected Cherenkov photons lead to a better time resolution compared to only one detected photon without crosstalk. The trend that SPCTR is better than the CTR was also observed in Dolenec et al (2016a) in the case of low photon number. 


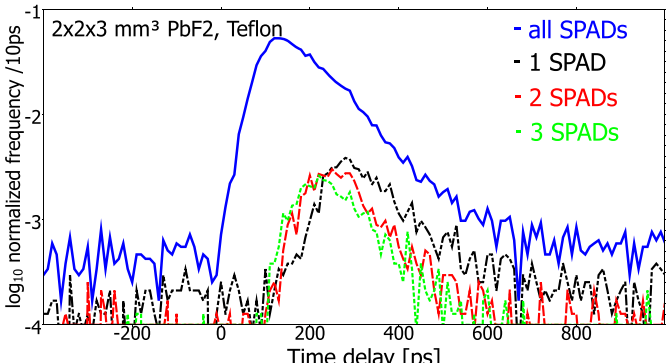

$2 \times 2 \times 3 \mathrm{~mm}^{3} \mathrm{PbF} 2$, Teflon
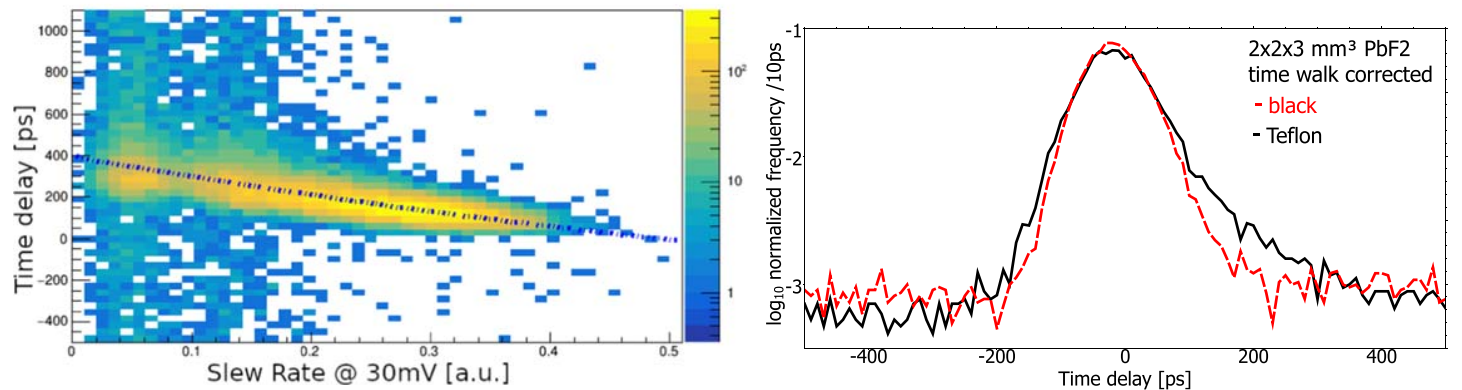

Figure 5. (top left) Time delay distribution for one (black), two (red), three (green) and all triggered SPADs (blue) for Teflon wrapped $2 \times 2 \times 3 \mathrm{~mm}^{3}$ crystals. (top right) Time delay distribution for black painted crystals. (bottom left) Scattered plot of the slew rate against the time delay with shown fit function used for time walk correction in the case of Teflon wrapped crystal. (bottom right) Time delay distribution for all triggered SPADs, after time walk correction, performed for both, the black and Teflon configurations.
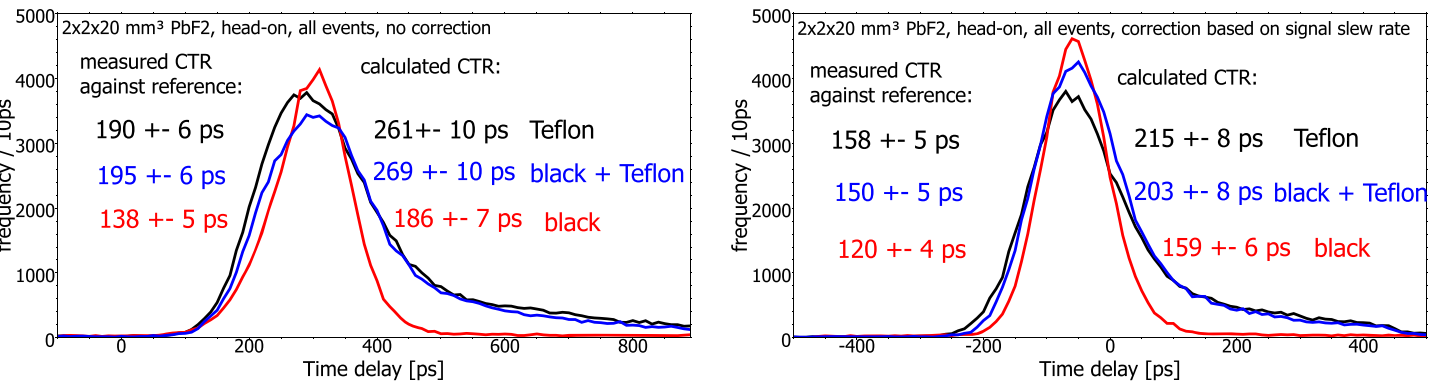

Figure 6. Time delay distribution before (left) and after time walk correction (right) for $20 \mathrm{~mm}$ long $\mathrm{PbF}_{2} \mathrm{crystals}_{\text {with different }}$ surface conditions. The measurement time was 200 minutes each and photopeak selection on the reference detector was performed.

Table 4. SPCTR, CTR before/after time walk correction (FWHM and FWTM) and detection efficiency corrected CTR [ps] accounting for not detected photons upon gamma interaction for various $\mathrm{PbF}_{2}$ crystal configurations. Energy ranges for LSO:CeCa (Teflon) and BGO (Teflon) are used without SiPM saturation considerations. Values for BGO are taken from (Kratochwil et al 2020 a). Errors are $\approx 4 \%$ of the values (up to $10 \%$ for det. $\overline{C T R}$ due to error on $\mu$ ). The leading edge threshold was set to $10 \mathrm{mV}$ for all the measurements (LSO $20 \mathrm{mV}$ ) which is close to the optimal settings.

\begin{tabular}{lcccccc}
\hline $\begin{array}{l}\text { Surface condition } \\
\text { or Material }\end{array}$ & $\begin{array}{c}\text { Geometry } \\
\left(\mathrm{mm}^{3}\right)\end{array}$ & $\begin{array}{c}\text { SPCTR } \\
\text { FWHM }\end{array}$ & $\begin{array}{c}\text { CTR } \\
\text { FWHM }\end{array}$ & $\begin{array}{c}\text { CTR cor } \\
\text { FWHM }\end{array}$ & $\begin{array}{c}\text { CTR cor } \\
\text { FWTM }\end{array}$ & $\begin{array}{c}\text { det. CTR } \\
\text { FWHM }\end{array}$ \\
\hline Teflon & $2 \times 2 \times 3$ & 166 & 190 & 142 & 293 & 184 \\
Bare & $2 \times 2 \times 3$ & 132 & 214 & 132 & 277 & 186 \\
Black+Teflon & $2 \times 2 \times 3$ & 173 & 230 & 159 & 348 & 234 \\
Black & $2 \times 2 \times 3$ & 113 & 235 & 134 & 269 & 514 \\
Teflon & $2 \times 2 \times 20$ & 263 & 261 & 215 & 582 & 290 \\
Black+Teflon & $2 \times 2 \times 20$ & 200 & 269 & 203 & 527 & 344 \\
Black & $2 \times 2 \times 20$ & 145 & 186 & 159 & 303 & 2252 \\
LSO [100-665 keV] & $2 \times 2 \times 3$ & $/$ & 142 & 123 & 224 & 123 \\
BGO [100-665 keV] & $2 \times 2 \times 3$ & $/$ & 238 & 193 & 567 & 193 \\
BGO [440-665 keV] & $2 \times 2 \times 3$ & $/$ & 169 & 151 & 331 & 151 \\
BGO [100-665 keV] & $2 \times 2 \times 20$ & $/$ & 334 & 298 & 1469 & 298 \\
BGO [440-665 keV] & $2 \times 2 \times 20$ & $/$ & 288 & 258 & 891 & 258 \\
\hline
\end{tabular}




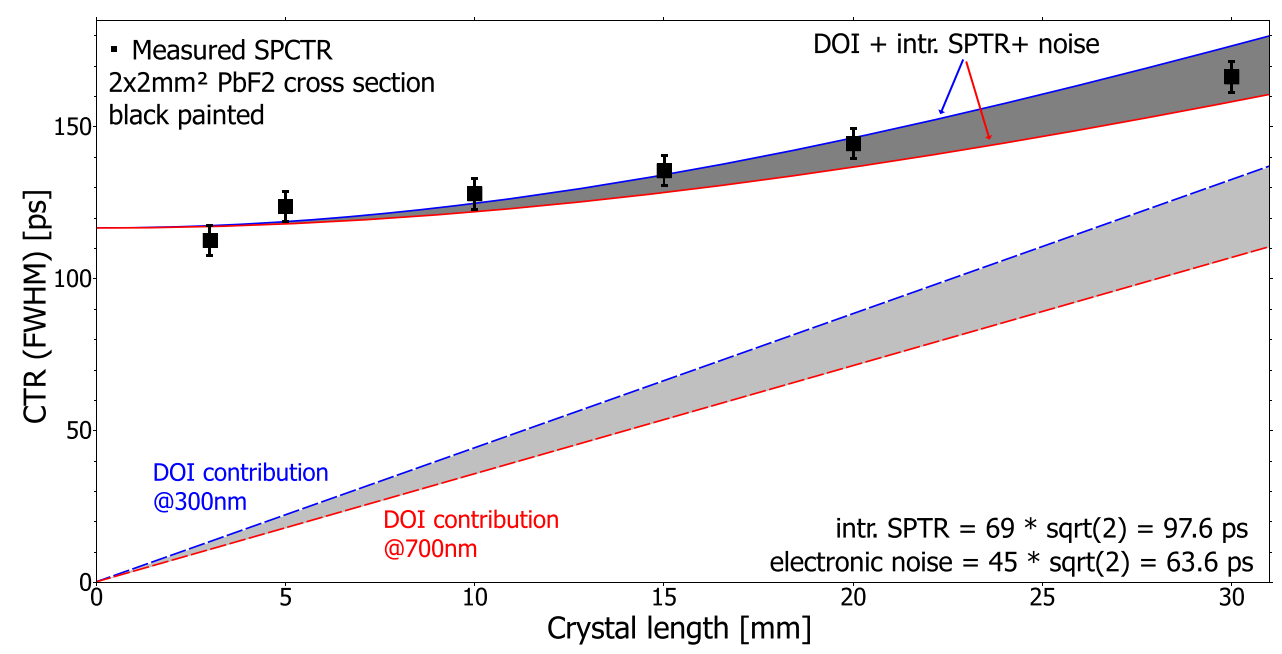

Figure 7. Contribution of the time resolution in the case of one detected photon for black painted crystals. (Dotted line:) Contribution of the crystal length due to uncertainty of depth of interaction for high $(n=1.9366 @ 300 \mathrm{~nm})$ and low refractive index ( $n=1.755$ @ $700 \mathrm{~nm}$ ). (Solid line:) Quadratically added contribution of electronic noise (2.35 $\cdot$ RMS of noisefloor $\left./ \frac{d V}{d t}\right)$, SPTR and DOI. (Black points:) Measured SPCTR.

The detection efficiency corrected CTR is normalized based on the detection probability $P_{\text {coinc }}$ from equation (4) and table 3 (det. $\left.\overline{C T R}=\mathrm{CTR}_{\text {cor }} / P_{\text {coinc }}\right)$ in order to compare the results with each other in terms of effective sensitivity. For standard scintillators having high light output both quantities are the same, since there are always photons detected upon gamma interaction in the crystal. In the case of sole Cherenkov radiators reducing the PTS by loosing photons inevitably lead to an overall drop of PET detector performance by much lower effective sensitivity (Schaart et al 2021).

\subsection{Intrinsic CTR limits for one detected photon}

While black painted crystals are most likely not suited for PET given the low sensitivity and therefore high $\operatorname{det} . \overline{C T R}$, they provide interesting features to study the contributions of measured time resolution. The case of one triggered SPAD is a crosstalk-free environment where light transfer efficiency (LTE) and PDE do not matter and the PTS is only coming from different DOIs and a small smearing due to photon propagation in the crystal (see section 4.4).

The measured SPCTR for $2 \times 2 \times 3 \mathrm{~mm}^{3}$ black painted crystals is solely determined by the SiPM properties: the intrinsic SPTR of the used SIPM is $69 \pm 6$ ps FWHM (Gundacker et al 2020a) with an already subtracted electronic noise contribution of $45 \pm 5 \mathrm{ps}$. Adding these two contributions in quadrature and multiplying with $\sqrt{2}$ for a coincidence condition, the expected SPCTR is $116.5 \pm 11.5 \mathrm{ps}$ FWHM, which is in good agreement with the measured value of $112.7 \pm 4.5 \mathrm{ps}$. Small differences can come from illuminating only a $2 \times 2 \mathrm{~mm}^{2}$ SiPM cross section compared to all the SiPM area which improves the SPTR (Nemallapudi et al 2016) and that the photon emission is not only at a fixed wavelength but continuous (Piull et al 2012).

SPCTR measurements with different crystal lengths were performed to study the impact of DOI on the timing resolution. Analytic models on DOI-induced time bias exist for standard scintillators (Toussaint et al 2019, Loignon-Houle et al 2020a) where the light transport and rise and decay time lead to additional uncertainty and in Cates et al (2015) DOI contribution has been shown of not being Gaussian like. In figure 7 the DOI bias was modeled by taking the time difference of different velocities of the gamma and optical photons at the extreme cases (small and large DOIs). This is certainly a very simple approximation, as no weighting for the DOIs was used. Due to crystal attenuation certain DOIs are more probable to interact, while photons produced at short DOIs far away from the SiPM need to travel longer and are more likely lost. Also the contribution of the PTS on top of the SPTR was assumed to be Gaussian like, which might only be valid for small DOI contribution with respect to the SPTR. For black painted crystals the approximation of the speed of the optical photons $(v=c / n)$ matches the data, since mainly direct photons are detected. Even when having very high number of prompt photons this intrinsic limit cannot be surpassed without accessing the DOI information.

To confirm that the SPCTR is not affected by the used material in case of Black surface, time resolution of a $2 \times 2 \times 4 \mathrm{~mm}^{3}$ Quartz glass was measured. While having a high floor of dark count events since the stopping power of Quartz is poor, a comparable time resolution of $123 \pm 7 \mathrm{ps} \mathrm{FWHM}$ as for $\mathrm{PbF}_{2}$ was measured. The small deterioration with respect to $\mathrm{PbF}_{2}$ might be due to enhanced reflections, as the Quartz was depolished, while the additional millimeter crystal length should only contribute to around $0.5 \mathrm{ps}$ and is negligible. 

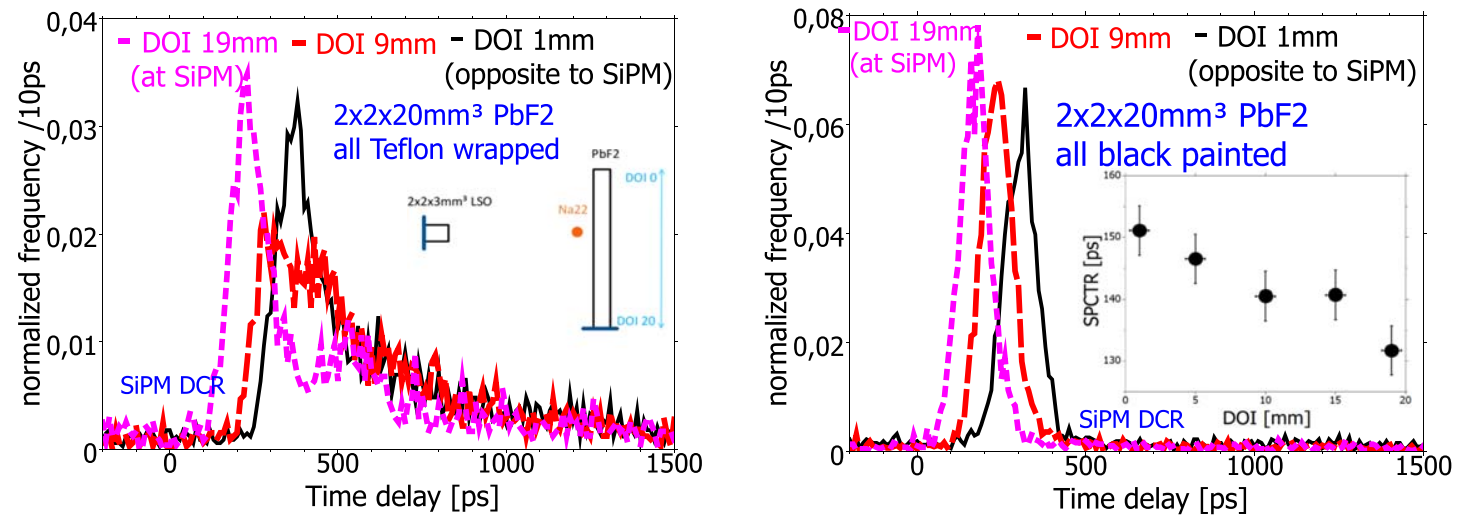

Figure 8. (left) Coincidence time delay between the reference crystal and Teflon wrapped $\mathrm{PbF}_{2}$ crystal. Inside the figure shows an illustration of the setup. (right) Coincidence time delay for black painted crystal, inside is the measured SPCTR (FWHM) for all DOI positions.
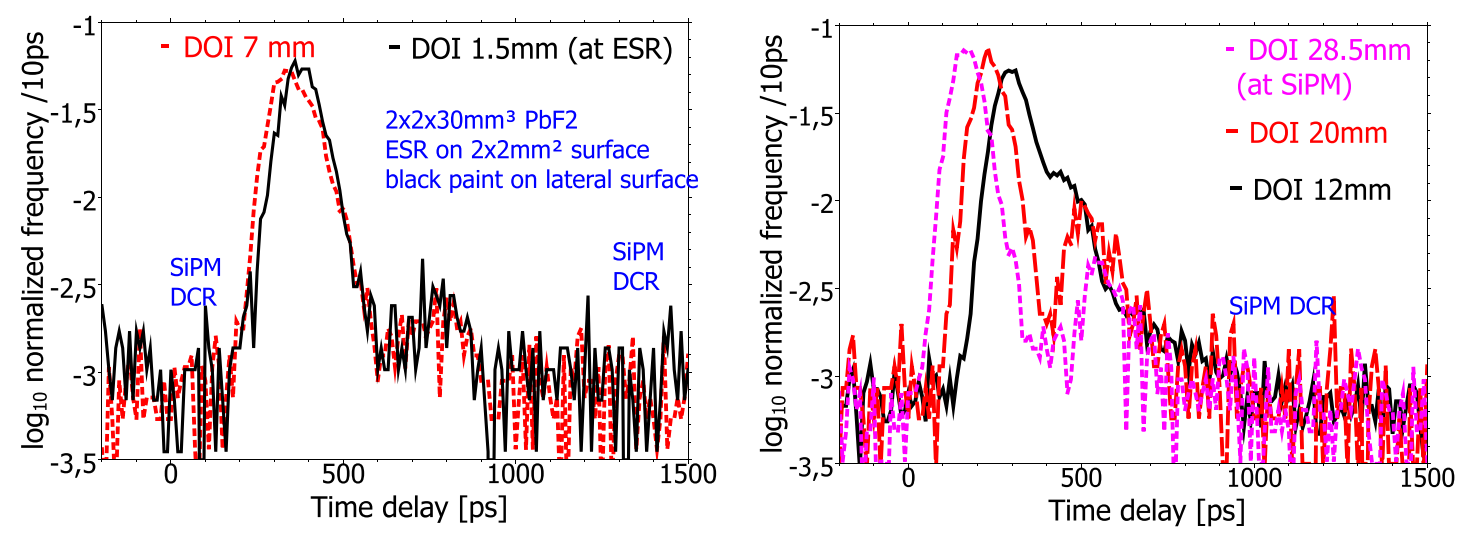

Figure 9. Coincidence time delay for $30 \mathrm{~mm}$ long $\mathrm{PbF}_{2}$ crystal. The lateral $2 \times 30 \mathrm{~mm}^{2}$ sides were black painted while ESR was placed on the $2 \times 2 \mathrm{~mm}^{2}$ side opposite to the SiPM. (left) Small DOI position. (right) Large DOI position. The ratio of the areas between the initial and reflected photon wave decreases from $2.3(\mathrm{DOI}=12 \mathrm{~mm})$ to $5.6(\mathrm{DOI}=20 \mathrm{~mm})$ and $13.2(\mathrm{DOI}=28.5 \mathrm{~mm})$ due to a high attenuation in this specific configuration.

\subsection{DOI collimated results for one detected photon}

To further evaluate the photon time propagation in the crystal, DOI collimated measurements were performed. The DOI setup is shown in the inset of figure 8, left. Depending on the experimental conditions, the photon can directly travel toward the SiPM or travel away from it being later reflected at the opposite crystal face leading eventually to its detection. Figure 8 shows the coincidence time delay histogram for two surface conditions: on the left for Teflon and on the right for Black. In the second case no reflections are visible, and only photons propagating directly to the SiPM contribute to the SPCTR. The center of the distribution is moving, since by changing the DOI position the distance of the two SiPMs is changing as well. The measured SPCTR slightly deteriorates from $132 \pm 4$ ps to $150 \pm 4$ ps when decreasing the DOI due to a larger dispersion of the photon paths (shown as inset in figure 8 , right). When the crystal is fully wrapped in Teflon photons can bounce between the surfaces and are kept longer in the crystal, largely increasing the variation of the travel time and therefore the SPCTR. In the case of interaction close to the SiPM one can identify the wave of photons which are backreflected at the end of the crystal and reach the SiPM at later times. This propagation of photons in high aspect ratio crystals is well understood and simulations are shown for example in Gundacker et al (2013), but to the authors knowledge have never been experimentally visualized. In the case of BGO (Kratochwil et al 2020c) and also LSO (Loignon-Houle et al 2020b) the CTR is better at short DOI positions, as the two waves of photons reach the SiPM at the same time, effectively increasing the initial photon time density. Work is ongoing to use this effect for extracting DOI information (Loignon-Houle et al 2020b). The distributions were normalized based on the total number of valid coincidences, since, in particular for the black painted configuration, a strong attenuation was observed.

In order to better visualize the time structure caused by the reflections opposite to the SiPM while keeping the time spread small, another DOI measurement for a $30 \mathrm{~mm}$ long $\mathrm{PbF}_{2}$ crystal was performed. Here the small 
$2 \times 2 \mathrm{~mm}^{2}$ face opposite to the SiPM was covered by ESR for mirror-like reflections. The four lateral sides were black painted to suppress reflections and time dispersion of the photon wave. Five different DOI positions were measured and to better discuss the results they are split in figure 9. For shallow DOI positions the back reflected wave is not or only barely visible, as the difference of travel distance of the two photon waves is too little to be resolved. Only faintly visible is a second peak being about 350 ps delayed with respect to the main peak coming from Fresnel reflections at the interface between crystal, Meltmount and SiPM. The reflections coming from the ESR are well visible on the right of figure 9 for DOI positions at 20 and $28.5 \mathrm{~mm}$ close to the SiPM. The abundance of measured reflected photons largely decreases with longer DOI positions, as the black paint leads to a short effective attenuation length. The measured time differences for figure 9 are all, within the experimental resolution, in excellent agreement with DOI positions and the speed of the photons inside the crystal $(n / c$ $\approx 6.5 \mathrm{ps} / \mathrm{mm}$ at $300 \mathrm{~nm}$ ). 


\section{Discussion on the results}

\subsection{Impact of crosstalk and correction methods on the time resolution}

In section 4 and figure 5, time resolution for one and more triggered SPADs are shown. Naively it is expected that more triggered SPADs lead to better time resolution, as more photons are detected. In this high crosstalk environment however, most events populating two triggered SPADs are caused by one detected photon and one crosstalk event. Those events do not carry better time information than the case of having only one triggered SPAD.

On top of this, the crosstalk introduces an additional time jitter. Depending when crosstalk happens the signal reaches the leading edge threshold earlier or later. Triggering on even smaller thresholds (e.g. $5 \mathrm{mV}$ instead of $10 \mathrm{mV}$ ) leads to better time resolution for most events since crosstalk has less time to propagate, but worse timing for one SPAD events due to a higher electronic noise contribution, since the slew rate $(\mathrm{d} V / \mathrm{d} t)$ at lower threshold generally is smaller. This is also the reason why time walk correction is so crucial, as to some extent it can correct the time walk caused by crosstalk.

For $20 \mathrm{~mm}$ long Black crystals using all events a CTR of $186 \pm 7$ ps FWHM without correction was calculated, compared to $174 \pm 7$ ps for a correction based on the measured amplitude, $177 \pm 7$ ps based on the charge, $180 \pm 7$ ps based on the signal rise time between 5 and $20 \mathrm{mV}$ and $159 \pm 6$ ps based on the slew rate at $30 \mathrm{mV}$ (see table 4). A correction based on the signal rise time at higher values (e.g. 20-60 mV) lead to similar time resolutions as the slew rate correction at $30 \mathrm{mV}$, but does not work for one-SPAD events since the signal amplitude is too low to pass the second threshold. Classical correction methods (e.g. based on the amplitude or integrated charge) are not that effective, as these quantities consider all the triggered SPADs even after the leading edge threshold. As a result the measured amplitude is only weakly correlated with the time walk, while the slew rate has a stronger dependency.

In the case of HF electronics, we have shown that corrections based on the slope of the signal (slew rate, rise time) will outperform corrections based on the amplitude or charge, demonstrated in figure 10 for two-SPAD events. Only when selecting on a high number of triggered SPADs it is more likely that two or more "real" Cherenkov photons are responsible for those events. In this case they carry improved time information, which allows to overcome the SPCTR limit. For example, sub-100 ps (99 \pm 4 ps FWHM, $191 \pm 6$ ps FWTM) CTR was measured for $2 \times 2 \times 3 \mathrm{~mm}^{3}$ long Teflon wrapped $\mathrm{PbF}_{2}$ crystals for $21 \%$ of all events when selecting on the highest slew rate $(4.3 \%$ in coincidence).

The most simple case to be considered is a two SPAD event, as in this case either two real photons are detected or only one photon with one crosstalk. When looking at the distribution of the slew rate for those events (filtering two-SPAD events based on the charge), a structure is visible, shown on the left of figure 10. Selecting only on certain slew rates of the two-SPAD events the center of the time delay distribution is moving illustrated on the right of figure 10 and the measured CTR is improving compared to all two-SPAD events. These results confirm the importance of the time walk correction based on the slew rate and might indicate towards an identification of crosstalk events compared to two real photon events. More detailed measurements with different light intensities (e.g. with laser) are required for validation.

\subsection{Intrinsic Cherenkov photon yield}

The intrinsic Cherenkov photon yield for an electron with a given speed can directly be calculated using the Frank-Tamm-formular (Leo 1994, Brunner et al 2014). Uncertainties arise, since recoil electrons not always have the same energy. For photo ionization on average 20 optical Cherenkov photons were simulated for $\mathrm{PbF}_{2}$ (Canot et al 2019) (above $250 \mathrm{~nm}$ ), while for BGO which has higher refractive index, $17 \pm 3$ Cherenkov photons were experimentally measured between 300 and $800 \mathrm{~nm}$ (Gundacker et al 2020a).

The measured number of Cherenkov photons stated in table 3 for small Teflon wrapped crystals is $\mu=2.08 \pm 0.21$ including crosstalk and $\mu=2.28$ without crosstalk considerations and a worse robust fit function. To estimate the intrinsic Cherenkov photon yield several correction factors need to be included: LTE in the crystal ( $c_{L T E} \approx 0.6$ ) (Gundacker et al 2020a), weighted PDE starting at $300 \mathrm{~nm}\left(c_{P D E} \approx 39 \%\right.$ ) (Gundacker et al 2020a), and correction factors regarding energy $c_{\text {energy }}=0.7$ and Cherenkov emission in the deep UV $c_{V U V}=0.77$. The correction on the deposited energy $c_{\text {energy }}$ is needed, since in the experiment both photoabsorption and Compton events were considered, where for the latter the Cherenkov photon yield is lower. The value was derived from Geant 4 simulations on a $2 \times 2 \times 3 \mathrm{~mm}^{3}$ BGO crystal comparing the intrinsic Cherenkov photon yield with $511 \mathrm{keV} \gamma$-irradiation for deposited energies above $100 \mathrm{keV}$ and for only $511 \mathrm{keV}$ deposition (Terragni 2020). $\mathrm{PbF}_{2}$ is transparent down to $250 \mathrm{~nm}$, while in the measurement Cherenkov photons between 250 and $300 \mathrm{~nm}$ were not detected due to the non-transparent optical glue and non-VUV sensitive SiPMs. The correction factor $c_{V U V}$ was calculated by comparing the integral of the $1 / \lambda^{2}$ Cherenkov distribution between 250-900 $\mathrm{nm}$ and 300-900 $\mathrm{nm}$. 

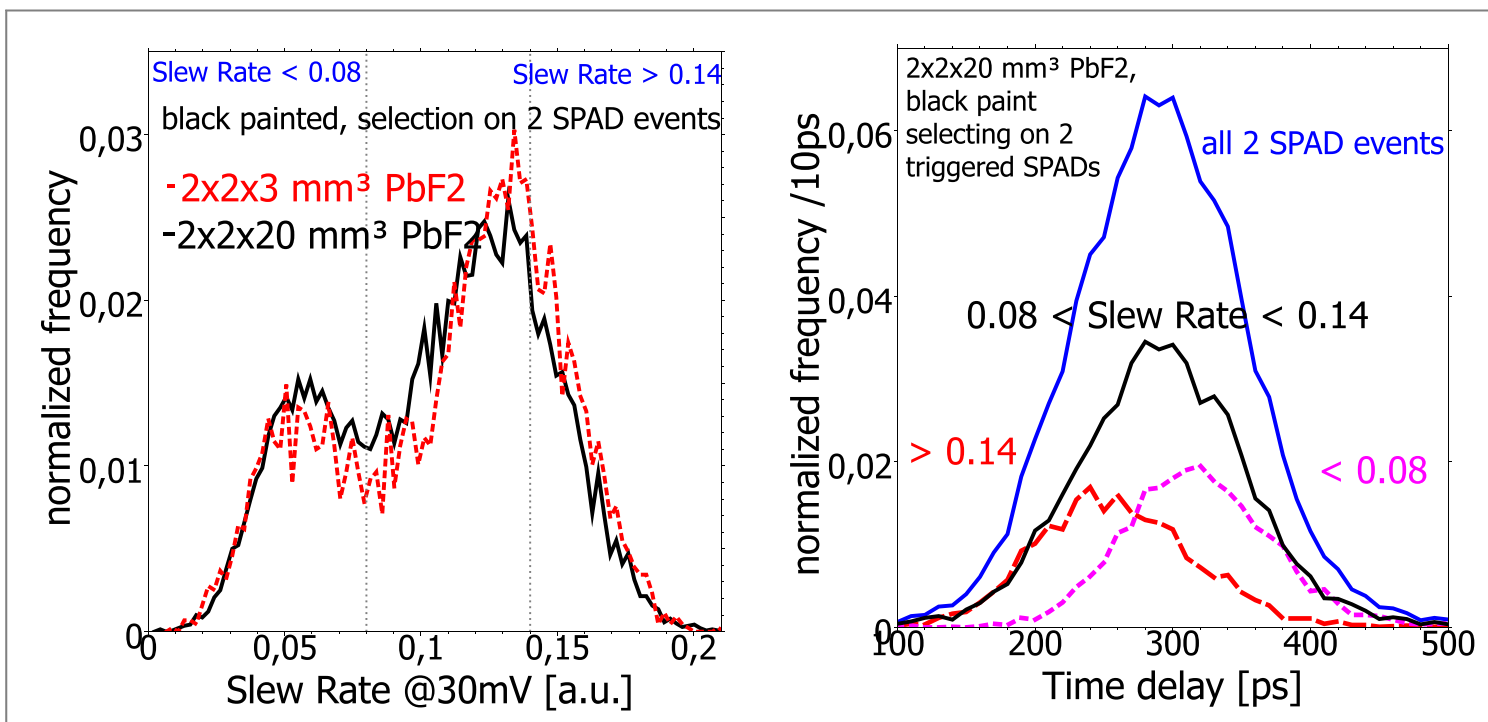

Figure 10. (left) Normalized histogram of the slew rate for 3 (red dotted) and $20 \mathrm{~mm}$ (black solid) long black painted $\mathrm{PbF}_{2} \mathrm{crystals}$ selecting on 2 triggered SPADs only. (right) Coincidence time delay distribution for $20 \mathrm{~mm}$ long black painted crystals using all 2 SPAD events (blue), events with high (red), average (black) and low (magenta) slew rate.

Including all the correction factors the intrinsic Cherenkov photon yield upon $511 \mathrm{keV}$ gamma interaction for $\mathrm{PbF}_{2}$ between 250 and $900 \mathrm{~nm}$ was calculated according to equation (5).

$$
I L Y_{P b F_{2}}=\mu \cdot \frac{1}{c_{L T E}} \cdot \frac{1}{c_{P D E}} \cdot \frac{1}{c_{\text {energy }}} \cdot \frac{1}{c_{V U V}}=16.5 \pm 3.3 .
$$

In the case of Teflon only around $20 \%$ of the data was used for the Cherenkov photon yield calculations, since for many events the number of triggered SPADs was exceeding the used range of the oscilloscope giving an additional uncertainty. Repeating the calculation for different SiPM overvoltage does not change the calculated value significantly, e.g. $\mu=1.81(38 \mathrm{~V}), \mu=1.75(37 \mathrm{~V}), \mu=1.88(36 \mathrm{~V}), \mu=1.86(35 \mathrm{~V}), \mu=1.82(34 \mathrm{~V})$ was calculated for the bare crystal. Thorough measurements with different crystal volumes and better dynamic acquisition ranges together with simulations are required for a more precise estimation. 


\section{Applicability of sole Cherenkov radiators with SiPMs at the system level}

Throughout this work, measurements in a highly optimized setup have been presented. For a detector consisting of thousands of channels it will not be applicable to grant a power consumption of more than $500 \mathrm{~mW}$ per channel. The same argument can be made for the acquisition system, as digitization via $3.5 \mathrm{GHz}$ bandwidth oscilloscope will not be possible on a system level. In this section we discuss challenges at a system level and provide a reflection for future R\&D for tracing the route toward a sole Cherenkov PET with SiPMs.

\subsection{Time resolution aspects}

The measured CTR values scale close to the analytic calculation considering DOI and SPTR impact. Even for $20 \mathrm{~mm}$ long crystals wrapped in Teflon, the contribution of the PTS is below 200 ps FWHM. As a result literature values on measured SiPM SPTR values with different acquisition systems can be reviewed to estimate the timing performance for Cherenkov radiators.

Using a NINO (Anghinolfi et al 2004) chip, SPTR values of $94 \pm 5$ ps FWHM for $1 \times 1 \mathrm{~mm}^{2}(175 \pm 7$ ps for $3 \times 3 \mathrm{~mm}^{2}$ ) SiPM size have been measured in Nemallapudi et al (2016) with FBK NUV SiPMs, which translates to 133 ps (247ps) CTR for two detectors in coincidence. Using the same SiPMs as in this work $\left(4 \times 4 \mathrm{~mm}^{2} \mathrm{FBK}\right.$ NUV-HD) and NINO chip, about 130 ps FWHM have been measured with this ASIC (Gundacker et al 2019). For SiPMs from Hamamatsu (S13360-3050PE), SPTR values of 214 ps with FlexTOT v2 (Sarasola et al 2017), and 176 ps FWHM with FASTIC ASIC (Sanchez and Ballabriga 2021) have been reported, compared to the estimated intrinsic SPTR value of $135 \pm 8$ ps FWHM (Gundacker et al 2019).

Another solution might be the use of digital SiPMs (Liu et al 2016), and to use the timestamp from the first triggered SPAD. For instance using $3 \times 3 \times 20 \mathrm{~mm}^{3} \mathrm{BGO}$ crystals and utilizing the Cherenkov emission, CTR values down to 330 ps FWHM have been reported (Brunner and Schaart 2017). Based on values presented in table 4, the time resolution should improve when replacing $\mathrm{BGO}$ with $\mathrm{PbF}_{2}$.

\subsection{Dark count events and signal recovery}

One major drawback of SiPMs are dark count events, which lead to a indistinguishable signal from the detection of few Cherenkov photons. DCR events are not correlated, so the probability of triggering two SPADs simultaneously is negligible. However, crosstalk can cause two or more SPADs to be triggered simultaneously, as described in equation (1). The probability distribution of detecting a noise event for a given crosstalk probability as function of the trigger threshold is shown on the left of figure 11 . When increasing the trigger threshold, it becomes at the same time less likely to detect a real event coming from Cherenkov photons after $\gamma$-interaction in the crystal. This drop of detection probability is illustrated on the right of figure 11 . This analytic calculations are derived from equations (1) and (2). When on average a high number of Cherenkov photons are detected and for low crosstalk, DCR events can successfully be suppressed by increasing the trigger threshold to 2 or more triggered SPADs without large loss of detection efficiency. Thanks to the excellent stopping power and high sensitivity of $\mathrm{PbF}_{2}$, such a selection will not harm the overall sensitivity too much, compared to less dense materials.

\subsection{Energy resolution}

Sufficiently good energy resolution in PET is required to reject detected gammas which scatter in the body and loose some energy, as such events would otherwise lead to misplaced line of responses. In the case of $\mathrm{PbF}_{2}$ or other pure Cherenkov radiators (ignoring novel concepts like Cherenkov charge-induction (Arino-Estrada et al 2019)) it is not possible to extract the energy of the gamma at all. However, the Cherenkov production process intrinsically provides a lower energy threshold. Based on simulations (Canot et al 2019) most of the $511 \mathrm{keV} \gamma$ interactions lead to an electron with kinetic energy of $423 \mathrm{keV}$ with side peaks at 495 and $507 \mathrm{keV}$ coming from the $\mathrm{K}, \mathrm{L}$ and $\mathrm{M}$-shell photo ionization of lead. If the interacting $\gamma$ has lower energy it results in further lower energy of the recoil electron. An electron with kinetic energy of $200 \mathrm{keV}$ will produce on average about 4 times lower number of Cherenkov photons compared to a kinetic energy of $400 \mathrm{keV}$ (Canot et al 2019). Hence gammas which are scattered in the body having less energy, produce fewer Cherenkov photons and are therefore less likely detected compared to non scattered gammas. This can be seen as different value of $\mu$ depending on the deposited energy.

Using equation (2) the detection probability for different values of $\mu$ can be analytically calculated. In figure $12 \lambda$ was fixed, while $\mu$ was modified depending on the deposited energy. The conversion of energy to mean number of detected Cherenkov photons depends on the crystal configuration and the SiPM, while the nonlinear Cherenkov photon yield was approximated based on simulations. Lastly the cutoff energy, where no Cherenkov photons are produced $(\mu=0)$ is around $100 \mathrm{keV}$, giving an additional filter for highly scattered gammas. 

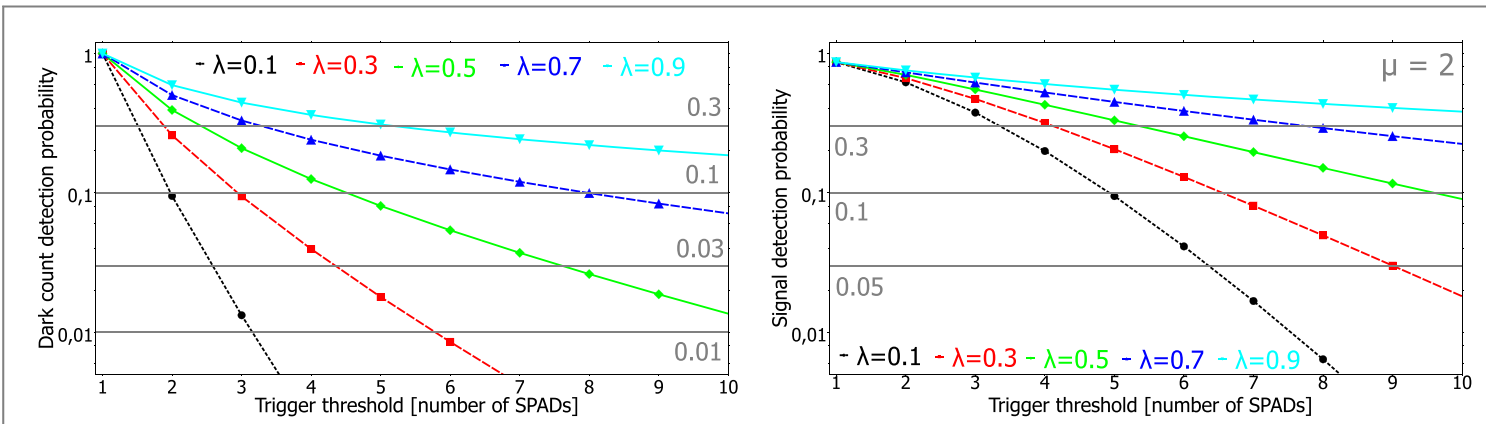

Figure 11. (left) Probability of detecting a dark noise event due to crosstalk as function of the applied threshold. (right) Probability of detecting a valid signal for $\mu=2$ for different crosstalk values as function of the selection threshold. Both plots are for a single detector and not in coincidence.

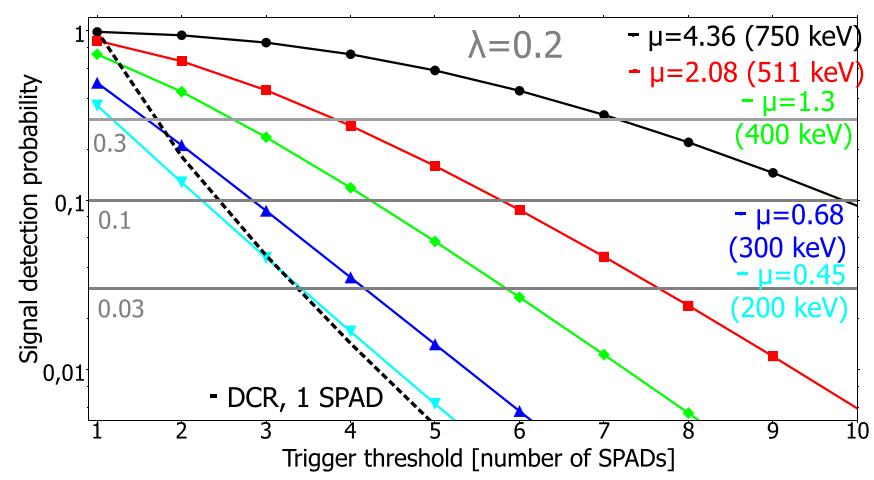

Figure 12. Probability of detecting a signal produced by a $\gamma$-interaction with variable energy as function of the threshold. The detection probability is for a single detector.

Both not-wanted types of events (DCR, scattered gammas) have on average lower number of triggered SPADs compared to true $511 \mathrm{keV}$ gamma interaction. To overcome the tradeoff between a "clean" signal (high threshold) and high statistics (low threshold), a weighted reconstruction could be an option where events with high number of triggered SPADs are given more priority or serve as a seed in the reconstruction process. A similar concept with a weighting based on different timing kernels for BGO was recently demonstrated (Efhimiou et al 2020). Overall, such a detector design is a completely new way of thinking (Efthimiou 2020), focusing mostly on cost and sensitivity, while energy resolution and scatter correction in the reconstruction are the weak points. Thorough simulation and image reconstruction for the case of sole Cherenkov radiators, including DCR background evaluating the feasibility of this approach are subject of future studies. 


\section{Outlook}

\subsection{SiPM improvements}

In this work only Cherenkov photons starting from $300 \mathrm{~nm}$ were used. With transparent optical coupling, as recently studied for $\mathrm{BaF}_{2}$ crystals (Pots et al 2020), and using SiPMs with extended detection efficiency in the VUV below $200 \mathrm{~nm}$ developed for liquid xenon (e.g. HPK-VUV S13370-3050CN or FBK-VUV (Capasso et al 2020, Gundacker et al 2020b)), more Cherenkov photons can be harvested giving a better time resolution and higher detection probability.

Further, there is a deterioration on time resolution due to crosstalk when having multiple SPADs triggered. Here the requirement is to reduce the crosstalk probability while keeping the good SPTR and PDE. Promising candidates for this are currently under development (Gola et al 2019) and subject for future work. A critical aspect in improving the time resolution significantly below $100 \mathrm{ps}$ is the SPTR of the photodetector. Here 3D integration (Nolet et al 2018) could be a viable solution with already reported SPTR values of 17.5 ps FWHM for a single SPAD including the time to digital converter. Also nano-structured light concentrators and nanophotonics (Enoch et al 2021) is an encouraging research line to improve both the SPTR and detection efficiency, while reducing crosstalk probability.

\subsection{Double-sided readout}

In the case of high aspect ratio crystals Cherenkov photons can travel towards or away from the SiPM, before being reflected and later detected by the photodetector. Unless the arrival time of the photons can be resolved in time, the second photon does not carry substantial time information, as it arrives too late to contribute to the development of the signal and time estimation. If the first photon is not detected, there is an increased time delay with respect to the time of interaction, responsible for the observed tail. Those problems can be solved when placing a second SiPM on the other side of the crystal. The measurement with the face black painted opposite to the SiPM imitates a double sided readout configuration having only one SiPM active. Here the SPCTR and also the time resolution after correction is better compared to having the crystal fully wrapped in Teflon, with lower number of detected Cherenkov photons. Depending on the requirement, a trigger in coincidence could also substantially decrease the DCR contribution without a large loss in sensitivity. Using double sided readout with twice as long (e.g. $30 \mathrm{~mm}$ ) crystals might be more favorable than having a normal sized crystal (e.g. $15 \mathrm{~mm}$ ) with only single sided readout, despite the same ratio of sensitive volume to readout channels.

Also light sharing (Pizzichemi et al 2019) might be an option, but special attention need to be given to false dark count events.

\subsection{Directionality}

Preserving some momentum of the incoming gamma photon with respect to the produced Cherenkov photons was expected due to Compton kinematics and from simulations. To the authors knowledge it is the first time that an experimental confirmation was given. For monolithic crystals and when covering all the crystal surface with SiPMs, which have an excellent detection efficiency close to 100\% (Somlai-Schweiger and Ziegler 2015), the recovery of the direction of the incoming gamma might be feasible, like for a Compton camera. This directionality might be also of interest for high energy particle detectors, as it gives another difference between scintillation and Cherenkov radiation besides the emission wavelength and scintillation kinetics for dual readout calorimetry (Lucchini et al 2013, Ferrari et al 2019).

\subsection{New method of SPTR measurements}

From an instrumental point of view the SPCTR can provide a new method for measuring the SPTR of a SiPM. This kind of measurements does not require a picosecond laser and can be also done with two SiPMs and two black painted $\mathrm{PbF}_{2}$ crystals in coincidence or one SiPM against a reference detector. The drawback is a lower count rate, hence longer measurement time and a large emission spectrum. However, the crystal does not necessarily have to be glued to the SiPM and an optical filter can be placed between the crystal and SiPM. Such a scheme would allow SPTR measurements at different wavelengths. Black painted $\mathrm{PbF}_{2}$ crystals can also be used for calibration purposes to extract the impulse response function for time correlated single photon counting measurements (Gundacker et al 2018, Martinazzoli et al 2020). 


\section{Conclusion}

In this work timing capabilities and Cherenkov photon yield of $\mathrm{PbF}_{2}$ read out by SiPMs was studied. The Cherenkov photon yield largely depends on the choice of surface treatment, while longer length is of secondary importance. The coincidence detection efficiency drops from $77 \%$ for $2 \times 2 \times 3 \mathrm{~mm}^{3}$ sized crystal and Teflon wrapping $\left(74 \%\right.$ for $\left.2 \times 2 \times 20 \mathrm{~mm}^{3}\right)$ to $26 \%(7 \%, 20 \mathrm{~mm})$ when the crystal surfaces are black painted. A directionality of the Cherenkov emission with respect to the incoming gamma is experimentally observed.

It has been demonstrated, that already one detected Cherenkov photon leads in all tested configurations to a decent time resolution, with an improvement when selecting on high number of triggered SPADs or performing time walk corrections. The SPCTR values for black painted crystals solely depend on the crystal geometry by having a contribution of the DOI plus the intrinsic timing properties of the SiPM together with the readout electronics. When wrapping with Teflon, both the CTR and the detection efficiency corrected CTR are comparable to timing values achieved with BGO. In this sense pure Cherenkov radiators coupled to SiPMs are a viable and cost effective alternative to current $\mathrm{L}(\mathrm{Y}) \mathrm{SO}$-based $\mathrm{PET}$ scanners with even better stopping power and photo fraction.

\section{Acknowledgments}

This work has been performed in the framework of the Crystal Clear Collaboration. The authors would like to thank Dominique Deyrail for his help in the crystal preparation and Alberto Gola (FBK) for providing the SiPM samples used in this study. Further the authors would like to express their highest gratitude to Francis LoignonHoule for interesting discussions on the Cherenkov-directionality and DOI bias and to Giulia Terragni on discussions regarding simulation work. 


\section{ORCID iDs}

Nicolaus Kratochwil (i) https://orcid.org/0000-0001-5297-1878

Stefan Gundacker ำ https://orcid.org/0000-0003-2087-3266

\section{References}

Acerbi F and Gundacker S 2019 Understanding and simulating SiPMs Nucl. Instrum. Methods Phys. Res. A 926 16-35

Anghinolfi F, Jarron P, Martemiyanov A N, Usenko E, Wenninger H, Williams M C S and Zichichi A 2004 NINO: an ultra-fast and lowpower front-end amplifier/discriminator AISC designed for the multigap resistive plate chamber Nucl. Instrum. Methods Phys. Res. A $533183-7$

Arino-Estrada G, Roncali E, Selfridge A R, Du J, Glodo J, Shah K S and Cherry S R 2021 Study of cerenkov light emission in the semiconductors TlBr and TlCl for TOF-PET IEEE Trans. Radiat. Plasma Med. Sci. 5 630-7

Arino-Estrada G, Kim H, Du J, Cirignano L J, Shah K S and Cherry S R 2020 Energy and electron drift time measurements in a pixel CCI TlBr detector with 1.3 MeV prompt-gammas Phys. Med. Biol. 66044001

Arino-Estrada G, Mitchell G S, Kim H, Du J, Kwon S I, Cirignano L J, Shah K S and Cherry S R 2019 First Cerenkov charge-induction (CCI) TlBr detector for TOF-PET and proton range verification Phys. Med. Biol. 64175001

Arino-Estrada G, Mitchell G S, Kwon S I, Du J, Kim H, Cirignano L J, Shah K S and Cherry S R 2018 Towards time-of-flight PET with a semiconductor detector Phys. Med. Biol. 63 04LT01

Berkson J 1980 Minimum chi-square, not maximum likelihood! Ann. Stat. 8 457-87

Brunner S E, Gruber L, Marton J, Suzuki K and Hirtl A 2014 Studies on the Cherenkov Effect for Improved Time Resolution of TOF-PET IEEE Trans. Nucl. Sci. $61443-7$

Brunner S E and Schaart D R 2017 BGO as a hybrid scintillator/Cherenkov radiatior for cost-effective time-of-flight PET Phys. Med. Biol. 624421

Canot C, Alokhina M, Abbon P, Bard J P, Breton D, Delagnes E, Maalmi J, Tauzin G, Yvon D and Sharyy V 2019 Fast and efficient detection of $511 \mathrm{keV}$ photons using Cherenkov light in $\mathrm{PbF}_{2}$ crystals, coupled to a MCP-PMT and SAMPIC digitization module JINST 14 P12001

Capasso M et al 2020 FBK VUV-sensitive Silicon Photomultipliers for cryogenic temperatures Nucl. Instrum. Methods Phys. Res. A 982 164478

Cates J W, Gundacker S, Auffray E, Lecoq P and Levin C S 2018 Improved single photon time resolution for analog SiPMs with front end readout that reduces influence of electronic noise Phys. Med. Biol. 63185022

Cates J W and Levin C S 2019 Electronics method to advance the coincidence time resolution with bismuth germanate Phys. Med. Biol. 64 175016

Cates J W, Vinke R and Levin C S 2015 Analytical calculation of the lower bound on timing resolution for pet scintillation detectors comprising high-aspect-ratio crystal elements Phys. Med. Biol. 605141

Consuegra D, Dolenec R, Korpar S, Krizan P and Pestotnik R 2019 Improving the cherenkov based pet performance using multi-layer detectors IEEE Symp. on Nuclear Science (NSS/MIC) (Manchester, UK, 26 October-2 November 2019) (Picastaway, NJ: IEEE) pp 1-4

Consuegra D, Korpar S, Krizan P, Pestotnik R, Razdevsek G and Dolenec R 2020 Simulation study to improve the performance of a wholebody $\mathrm{PbF}_{2}$ Cherenkov TOF-PET scanner Phys. Med. Biol. 65055013

Conti M and Bendriem B 2019 The new opportunities for high time resolution clinical TOF PET Clin. Transl. Imaging $7139-47$

Dolenec R, Korpar S, Krizan P and Pestotnik R 2015 Cherenkov TOF PET with silicon photomultipliers Nucl. Instrum. Methods Phys. Res. A $804127-31$

Dolenec R, Korpar S, Krizan P, Pestotnik R and Verdel N 2016a The performance of silicon photomultipliers in cherenkov TOF PET IEEE Trans. Nucl. Sci. $632478-81$

Dolenec R, Korpar S, Krizan P and Pestotnik R 2016b SiPM timing at low light intensities IEEE Nuclear Science Symp., Medical Image Conf. (NSS/MIC/RTSD) (Strasbourg, France, 29 October-6 November 2016) (Picastaway, NJ: IEEE) pp 1-5

Dolenec R, Korpar S, Krizan P, Pestotnik R, Stanovnik A and Verheyden R 2011 Time-of-flight measurements with Cherenkov photons produced by $511 \mathrm{keV}$ photons in lead crystals IEEE NSS/MIC TN (Knoxville, TN, 30 October-6 November 2010) (Picastaway, NJ: IEEE) pp 280-4

Efhimiou N, Kratochwil N, Gundacker S, Polesel A, Salomoni M, Auffray E and Pizzichemi M 2020 TOF-PET image reconstruction with multiple timing kernels applied on Cherenkov radiation in BGO IEEE Trans. Radiat. Plasma Med. Sci. 5703-11

Efthimiou N 2020 New Challenges for PET Image Reconstruction for Total-Body Imaging PET Clin. 15 453-61

Enoch S, Gola A, Lecoq P and Rivetti A 2021 Design considerations for a new generation of SiPMs with unprecedented timing resolution JINST 16 P02019

Ferrari R, Pezzotti L, Caccia M, Santoro R and Antonello M 2019 Dual-readout calorimetry Int. J. Mod. Phys. A 341940019

Gola A, Acerbi F, Capasso M, Marcante M, Mazzi A, Paternoster G, Piemonte C, Regazzoni V and Zorzi N 2019 NUV-sensitive silicon photomultiplier technologies developed at foundazione bruno kessler Sensors 19308

Gundacker S, Auffray E, Pauwels K and Lecoq P 2016a Measurement of intrinsic rise times for various L(Y)SO and LuAG scintillators with a general study of prompt photons to achieve 10 ps in TOF-PET Phys. Med. Biol. 612802

Gundacker S, Acerbi F, Auffray E, Ferri A, Gola A, Nemallapudi M V, Paternoster G, Piemonte C and Lecoq P 2016b State of the art iming in TOF-PET detectors with LuAG, GAGG and L(Y)SO scintillators of various sizes coupled to FBK-SiPMs JINST 11 P08008

Gundacker S and Heering A 2020 The silicon-photomultiplier: fundamentals and applications of a modern solid-state photon detector Phys. Med. Biol. 65 17TR01

Gundacker S, Knapitsch A, Auffray E, Jarron P, Meyer T and Lecoq P 2013 Time resolution deterioration with increasing crystal length in a TOF-PET system Nucl. Instrum. Methods Phys. Res. A 737 92-100

Gundacker S, Turtos R M, Auffray E and Lecoq P 2018 Precise rise and decay time measurements of inorganic scintillators by means of X-ray and $511 \mathrm{keV}$ excitation Nucl. Instrum. Methods Phys. Res. A 891 168-9002

Gundacker S, Turtos R M, Auffray E, Paganoni M and Lecoq P 2019 High-frequency SiPM readout advances measured coincidence time resolution limits in TOF-PET Phys. Med. Biol. 64055012

Gundacker S, Turtos R M, Kratochwil N, Pots R H, Paganoni M, Lecoq P and Auffray E 2020a Experimental time resolution limits of modern SiPMs and TOF-PET detectors exploring different scintillators and Cherenkov emission Phys. Med. Biol. 65025001 
Gundacker S et al 2020b VVacuum ultraviolet silicon photomultipliers applied to $\mathrm{BaF}_{2}$ cross-luminescence detection for high-rate ultrafast timing applications Phys. Med. Biol. 66114002

Kim H, Cirignano L, Churilov A, Ciampi G, Higgins W, Olschner F and Shah K 2009 Developing Larger TlBr Detectors- Detector Performance IEEE Trans. Nucl. Sci. 56 819-23

Kratochwil N, Gundacker S, Lecoq P and Auffray E 2020a Pushing Cherenkov PET with BGO via coincidence time resolution classification and correction Phys. Med. Biol. 65115004

Kratochwil N, Auffray E and Gundacker S 2020b Exploring Cherenkov emission of BGO for TOF-PET IEEE Trans. Radiat. Plasma Med. Sci. 5619-29

Kratochwil N, Auffray E and Gundacker S 2020c Exploring Cherenkov emission of BGO for multi kernel TOF-PET Oral presentation at IEEE Nuclear Science Symp. \& Medical Imaging Conf. (Boston, USA ) 05.11.2020

Lecoq $\mathrm{P}$ et al 2020 Roadmap toward the 10 ps time-of-flight PET challenge Phys. Med. Biol. 65 21RM01

Leo W R 1994 Techniques for Nuclear and Particle Physics Experiments (Berlin: Springer)

Liu Z, Gundacker S, Pizzichemi M, Ghezzi A, Auffray E, Lecoq P and Paganoni M 2016 In-depth study of single photon time resolution for the Philips digital silicon photomultiplier JINST 11 P06006

Loignon-Houle F, Toussaint M, Lee M S, Cates J W and Lecomte R 2020a Experimental validation of a coincidence time resolution metric including depth-of-interaction bias for TOF-PET Phys. Med. Biol. 65245004

Loignon-Houle F, Gundacker S, Toussaint M, Camirand Lemyre F, Auffray E, Fontaine R, Charlebois S A, Lecoq P and Lecomte R 2020b through signal arrival time distribution: A theoretical descripton including proof of concept measurements Phys. Med. Biol. 66095015

Lucchini M, Medvedeva T, Pauwels K, Tully C, Heering A, Dujardin C, Lebbou K, Lecoq P and Auffray E 2013 Test beam results with LuAG fibers for next-generation calorimeters JINST 8 P 10017

Lucchini M T, Chung W, Eno S C, Lai Y, Lucchini L, Nguyen M and Tully C G 2020 New perspectives on segmented crystal calorimeters for future colliders JINST 15 P11005

Martinazzoli L, Kratochwil N, Gundacker S and Auffray E 2020 Scintillation Characterization and State-of-the-art Timing Performance of $\mathrm{Gd}_{3} \mathrm{Al}_{2} \mathrm{Ga}_{3} \mathrm{O}_{12}$ Single Crystals Nucl. Instrum. Methods Phys. Res. A 1000165231

Murty R C 1965 Effective atomic numbers of heterogeneous materials Nature 207 398-9

Nemallapudi M V, Gundacker S, Lecoq P and Auffray E 2016 Single photon time resolution of state of the art SiPMs JINST 11 P10016

Nolet F, Dubois F, Roy N, Parent S, Lemaire W, Massie-Godon A, Charlebois S A, Fontaine J and Pratte J 2018 Digital SiPM channel integrated in CMOS $65 \mathrm{~nm}$ with 17.5 ps FWHM single photon timing resolution Nucl. Instrum. Methods Phys. Res. A 912 29-32

Ota R, Kamada K, Kim K J, Kochurikhin V V and Yoshikawa A 2020 Evaluation of $\mathrm{HfO}_{2}$ crystal as high-performance Cherenkov radiator Poster Presentation at IEEE Nuclear Science Symp. \& Medical Imaging Conf. vol 05 (Boston, USA) p 11

Ota R, Nakajima K, Ogawa I, Tamagawa Y, Shimoi H, Suyama M and Hasegawa T 2019 Coincidence time resolution of 30 ps FWHM using a pair of Cherenkov-radiator-integrated MCP-PMTs Phys. Med. Biol. $6407 \mathrm{LT} 01$

Ota R et al 2021 Lead-free MCP to improve coincidence time resolution and reduce direct interactions Phys. Med. Biol. 66064006

Piull V, Bazin C, Breton D, Burmistrov L, Chaumat V, Dinu N, Maalmi J, Vagnucci J F and Stocchi A 2012 Single photoelectron timing resolution of SiPM as a function of the bias voltage, the wavelength and the temperature Nucl. Instrum. Methods Phys. Res. A 695 354-8

Pizzichemi M, Polesel A, Stringhini G, Gundacker S, Lecoq P, Tavernier S, Paganoni M and Auffray E 2019 On light sharing TOFPET modules with depth of interaction and 157 ps FWHM coincidence time resolution Phys. Med. Biol. 64155008

Pots R H, Auffray E and Gundacker S 2020 Exploiting cross-luminescence in $\mathrm{BaF}_{2}$ for ultrafast timing applications using deep-ultraviolet sensitive HPK silicon photomultipliers Front. Phys. 8482

Roncali E, Kwon S I, Jan S, Berg E and Cherry S R 2019 Cerenkov light transport in scintillation crystals explained: realistic simulation with GATE Biomed. Phys. Eng. Express 5035033

Sanchez D and Ballabriga R 2021 Measurements on FastIC FastIC Users Meeting online presentation, 16.03.2021

Sarasola I, Nemallapudi M V, Gundacker S, Sanchez D, Gascon D, Rato P, Marin J and Auffray E 2017 A comparative study of the time performance between NINO and FlexTOT ASICs JINST 12 P04016

Schaart D R 2021 Physics and technology of time-of-flight PET detectors Phys. Med. Biol. 6609 TR01

Schaart D R, Schramm G, Nuyts J and Surti S 2021 Time of flight in perspective: instrumental and computational aspects of time resolution in positron emission tomography IEEE Trans. Radiat. Plasma Med. Sci. 5 598-618

Seifert S, van Dam H T and Schaart D R 2012 The lower bound on the timing resolution of scintillation detectors Phys. Med. Biol. 57 1797

Somlai-Schweiger I and Ziegler S I 2015 CHERENCUBE: Concept definition and implementation challenges of a Cherenkov-based detector block for PET Med. Phys. 42 1825-35

Tao L, He Y, Kanatzidis M G and Levin C S 2019 Study of the coincidence time resolution of new perovskite bulk crystals IEEE Nuclear Science Symp. and Medical Imaging Conf. (NSS/MIC) (Manchester, UK, 26 October-2 November 2019) (Picastaway, NJ: IEEE) pp 1-3

Terragni G 2020 Exploiting Cherenkov radiation in semiconductors for fast timing in PET Master Thesis University Milano-Bicocca, Italy

Toussaint M, Loignon-Houle F, Dussault J and Lecomte R 2019 Analytic model of DOI-induced time bias in ultra-fast scintillation detectors for TOF-PET Phys. Med. Biol. 64065009

Vinogradov S 2012 Analytical models of probability distribution and excess noise factor of solid state photomultiplier signals with crosstalk Nucl. Instrum. Methods Phys. Res. A $695247-51$

Vinogradov S 2018 Approximation of coincidence time resolution models of scintillator detectors with leading edge discrimination Nucl. Instrum. Methods Phys. Res. A 912 149-53 